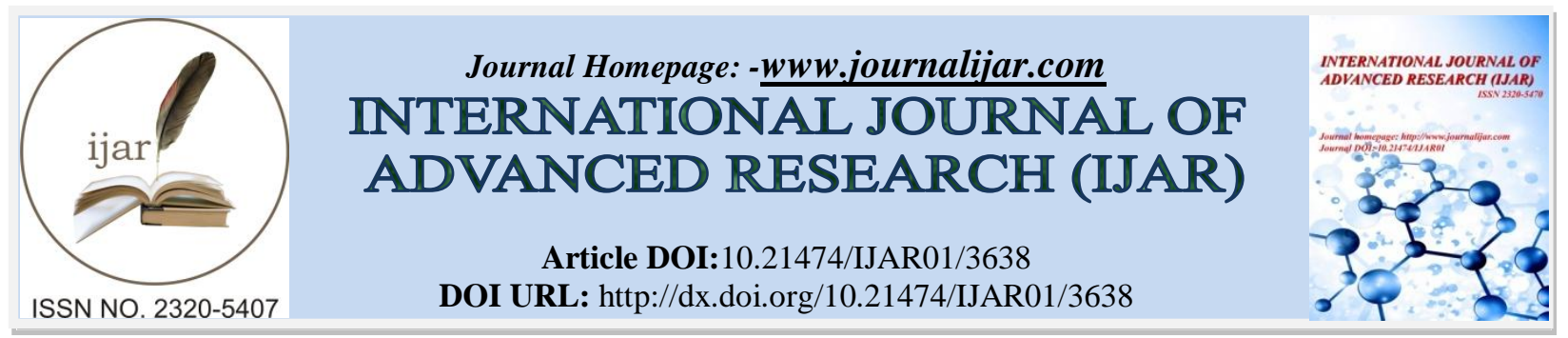

RESEARCH ARTICLE

\title{
GENETIC DIVERSITY AMONG FOUR MOMORDICA SPECIES USING RAPD, SSR AND ISSR MARKERS
}

\section{${ }^{*}$ Anjali Shukla ${ }^{1,2}$, D. P. Sinha ${ }^{1,3}$, D. R. Bhardwaj ${ }^{1}$, Abaidya Nath Singh ${ }^{4}$, Pankaj Kumar ${ }^{4}$ and Major Singh ${ }^{1}$.}

1. Divisionof CropImprovement,IndianInstituteofVegetableResearch,PostBox01,Jakhini (Shahanshahpur), Varanasi,UttarPradesh221 305,India.

2. Department of Biotechnology, Microtek College of Management and Technology, Varanasi.

3. NationalInstituteofVirology,C/oRGICDCampus,NearNimhans,Bangaluru,Karnataka-560 029,India.

4. Department of Botany, UdaiPratap Autonomous College, Varanasi.

\section{Manuscript Info}

\section{Manuscript History}

Received: 08 January 2017

Final Accepted: 07 February 2017

Published: March 2017

Key words

Bootstrapanalysis,Moleculardiversity, Momordica,Morphological characterization.

\section{Abstract}

Background: GenusMomordica, iswidely distributed overtropicandsubtropic region. Besidesthecultivatedbitter gourd(M.charantiaL.varcharantia), manyotherspeciesofthegenusoccurri nginthewildstatehavebeenfound inIndiaandneighboring countries.Amongthem, monoecious, M.charantiaandM.balsaminaanddio ecious, $M$.

dioicaandM.cochinchinensisexhibitdivergenceinmorphologicalcharacte rslikegrowthhabit,maturity, andfruit shapeandsize, sexexpression, leaf,rootandseedcharacters.

Methods: We studied the twentyfouraccessionsrepresentingfourMomordicaspeciesincludingtwogynoeci ouslinesof M.charantianamelyGy-323,Gy-333 by morphological and molecular analysis through RAPD, SSR and ISSR markers.

Results: The analysis of study based on dendrogram obtainedfromthedistancematrixfromthemeanvalueofthe

15quantitativetraitsforthegenotypesgroupedalltheaccessionsintotwomaj orclusters.

Onecluster separatestwodistinctgroupseachaccessionsofM.charantiaandM.cochinc hinensisandM.balsaminaformed distinctgroupwithina major clusterwhilethesecond major clusterconsistedofallaccession ofM.dioica.For $\quad$ moleculardiversityanalysis, $\quad 101$ primersincluding50RAPD, 16SSR and35ISSRprimersproducedatotal of600 scorable amplicons across fourspecies, ofwhich586(97.08\%) werepolymorphic. Seventeen markersdifferentiatingmonoecious anddioeciousand85-amplicons specifictooneofthefourMomordicaspecieswere

identified.TheUPGMAdendrogramobtainedfromJaccard'ssimilaritycoef ficient(averagesimilarityof0.38)

showedtwomajorclustersclearlydistinguishing monoeciousanddiociousspecieswithhighbootstrapvalue(100) betweennodes.

Conclusions: 
basedonquantitativetraitsandmol eculardiversityshowedsome correspondenceintheclusteringpattern

ofaccessionsrepresentingaspecies.Molecularmarkersweremoreefficienti ndifferentiatingtheaccessionsatandwithinspecies morphologicalmarkers. levelthan

Copy Right, IJAR, 2017,. All rights reserved.

\section{Introduction}

Bitter gourd(syn. bitter melon, Momordicacharantia L.), aneconomicallyimportant memberofthegenusMomordica(Family:Cucurbitaceae)iswidelycultivatedinIndia,China,Malaysia,AfricaandSouthAm erica(Raj et.al.1993;Singh 1990)foritsimmaturefruitsasvegetable. Bittergourdisalsousedasatraditionalmedicinefor diabetes in India,ChinaandCentralAmerica (Groveretal.2002;Yehetal.2003),vaccine (Duttaetal.1981)and otherhealthrelatedailmentsduetoitshealthpromotingsubstancessuchascharantin(Yehetal.2003).Thegenus

Momordicacomprising of59speciesiswidelydistributedallovertropicandsub-tropicregionbutthediversity of thespecies ismoreintropicalAfricaincludingseveralspeciesofeconomical andmedicinalimportancepossessing differentsexformanddifferentbasicchromosome number.Aboutathirdofthespeciesaremonoecious and remainingdioecious(Schaefer,2010). Theincompatibility among2n=2x=22,2n=2x=28and2n=4x=56speciesin thisgenusisindicatedbythenegativeresultsofcrossingsbetween M.charantiaL.andM.muricataL.and between M.dioicaRoxb.M.cochinchinensisSpreng.(TrivediandRoy1972).Besidesthecultivatedbittergourd( $M$.

charantiaL.varcharantia),severalspeciesarepresentinIndia,SriLanka,Bangladesh, $\quad$ Myanmar,Malay,etc. (Hooker1879)occurringinthewild stateandaregatheredbytribalcommunitiesasvegetables.Sevenspeciesoccur inIndiaandamongthem $M$.

charantiaandM.balsaminaaremonoeciouswhileM.dioica, andM.cochinchinensisaredioeciousspeciesandarepropagated vegetativelythroughtuberousroot(Rashid1993).Thesespeciesexhibit

morphologicaldivergenceincharacterslikegrowthhabit,maturity,fruitshapeandsize,sexexpression, leaf,root

andseedcharacters(RobinsonandDecker-Walters 1999). Thefruitsarebitterintasteandaredesirablefor consumption.Thus,M.charantiaisperhapsistheonly speciesinwhichbitternesshasbeen consideredasatraitfor selectionduringdomestication. Besidesmanyculinary usesinsouth,southeastandeastAsia,thegenusisalso cultivatedforitsuseasfolkmedicines.

M.charantiaprovides forrelativelybroad phenotypicvariation (Kundu 2012, Iqbal 2016), whichproduceslargefusiformfruits, whileM.dioicaisanimportantvegetableintheIndiansub-continent. Ithasmanyadvantages, likehigh marketprice,goodnutritionalvalueandlonger shelf life(Rasul2003, Singh 2013).Sweetgourd(M.cochinchinensis) occursinpeninsular region,humidtropicalforestsandalsoinnorth-eastern regionandbalsampearM.balsaminaoccursin north-western,IndoGangeticplainsadaptedtodrysandysoils.Sweetgourd,teaslegourdand balsampear beingminorvegetables.

Application ofmolecularmarkersastoolforidentificationandcharacterization efficientmanagementanduseinplantbreedinghasgainedimportance(Karp\&Edwards1997). Amongthe differenttypesofmolecularmarkers,randomamplifiedpolymorphicDNA(RAPD)(Williamsetal.,1990)is usefulfortheassessmentofgeneticdiversity(Ulanowskyetal.2001;Baranskietal.2001;Pradeepkumaretal. 2003;Caietal.2007;Tiwarietal.2009;).Thetechniqueisideallysuitedforfingerprintingapplicationsbecauseitis fast(Gwanamaetal.2000),requireslittlematerial(Loweetal.1996)andtechnicallyeasy(FenwickandWard 2001).Thewideavailabilityofcommercialprimersmakesthistechniqueswidespread (Gilliesetal. ofgermplasmfortheir inexpensiveandyieldslargenumbersofmarkers(MartinandBermejo2000).

Recently,someunderstanding ofthegeneticvariationshasbeenachievedusing transcriptome analysis of within M.charantiaspecies (Shukla et al. 2015) and molecularmarkerslike RAPD (Deyetal.2006; Beheraetal.2008;Bharthietal.2012), Inter SimpleSequenceRepeat (ISSR) (Singhetal.2007; Bharthietal.2012),AFLP (Gaikwadetal.2008)andSimpleSequenceRepeat (SSR)(Jietal.2012)inM.charantiaandinrelated $\operatorname{species}(M$. dioica) usingRAPD(Rasul etal.2007)andSSRmarkers(Jietal.2012).TransferabilityofSSRmarkershasalsobeenreportedinrelated 
species(Jietal.2012).Although,mostofthesestudieshavefocusedontheefficiencyofdifferentmolecular markersforassessingthediversitywithinonespecies, noneaimedatassessingthediversity Momordicaspecies.Thepresenteffortsaimtogetacomparativeaccountofmorphological amongsomerelated acrossthegenusandunambiguously identify differentspeciesofMomordicausingmolecularandmorphological markers.

\section{MaterialsandMethods \\ Plantmaterials}

Twentyfouraccessions selectedbasedonmorphological

offourMomordicaspeciescollectedfromdifferentagro-climaticzonesofIndiawere diversityandgeographicaldistributionandwereevaluatedattheResearchFarm, IndianInstituteofVegetableResearch,Varanasi,(U.P.)duringrainy-winter seasonof2014. Thelistofaccessions includedinthestudyisdetailedinTable 1.

Table 1: SalientFeaturesofusedMomordicagenotypes.

\begin{tabular}{|c|c|c|}
\hline Species & Source & SalientFeatures \\
\hline $\begin{array}{l}\text { Spinegourd(Momordica } \\
\text { charantia) }\end{array}$ & & $\begin{array}{l}\text { Monoecious,trailingvine withthinstemsandtendrils, Theleavesare heart- } \\
\text { shaped,5-10cmindiameter,cutinto5-7lobes,Flowersare bornesinglyinthe } \\
\text { leafaxils,fruitsaresmalltorare } \\
\text { fleshandbrightredplacentato } \\
\text { whichtheseedsareattached,Seedsaretanandoval,witharoughetched surface. }\end{array}$ \\
\hline GY-323 & IIVR & Gynoeciousplant,Largevines,largesizeleaveswithheartshape, \\
\hline GY333 & IIVR & Gynoeciousplant,mediumvines, morebranching,profusebearing,greenfruis \\
\hline DRAR-1 & TNAU & Monoecious, large vines,mediumleaves, lessbranching,lightgreenfruits \\
\hline VRBT-1 & ANGARU & $\begin{array}{l}\text { Monoecious, morebranching,mediumsizeleaves,continuousfruitingfor } \\
\text { longerperiod,greenfruits }\end{array}$ \\
\hline MC-84 & TNAU & Monoecious,mediumsizeleaves,lightgreenfruits,mediumsize fruits \\
\hline DVBTG-7 & IIVR & $\begin{array}{l}\text { Monoecious,mediumsizeleaves, morebranchinggreenfruits, mediumsize } \\
\text { fruits, }\end{array}$ \\
\hline DVBTG-5 & IIVR & $\begin{array}{l}\text { Monoecious, mediumsizeleaves, morebranchinggreenfruits, mediumsize } \\
\text { fruits, }\end{array}$ \\
\hline PDM & IARI & Monoecious,mediumsizeleaves,morebranchinggreenfruits,smallsize fruits, \\
\hline $\begin{array}{l}\text { Sweetgourd(Momordica } \\
\text { cochinchinensis) }\end{array}$ & & $\begin{array}{l}\text { Dioecious,rootsdevelopsbiggertubers, leavesarebigger,flowersarelarge, } \\
\text { yellow, } 3 \text { smalldeepblackorbluecirculardotsatthe baseofpetal,Flowerslarge } \\
\text { oblongfruits,fruitsarelightgreentoyellowin colour. }\end{array}$ \\
\hline RCSG-1 & $\begin{array}{l}\text { ICARRes. } \\
\text { Complex } \\
\text { Shilong }\end{array}$ & Dioecious, vigorousplant, lightgreenfruits,morefruiting \\
\hline RCSG-7 & $\begin{array}{l}\text { ICARRes. } \\
\text { Complex } \\
\text { Shilong }\end{array}$ & Dioecious, mediumplant, lightyellowfruits, mediumfruiting \\
\hline RCSG-5 & $\begin{array}{l}\text { ICARRes. } \\
\text { Complex } \\
\text { Shilong }\end{array}$ & Dioecious, vigorousplant,morespinyfruits, morefruiting,yellowfruits \\
\hline RCSG-36 & $\begin{array}{l}\text { ICARRes. } \\
\text { Complex } \\
\text { Shilong }\end{array}$ & Dioecious, vigorousplant,toughrind,less fruitinglightgreenfruiting \\
\hline DRMC-22 & $\begin{array}{l}\text { Kalayani } \\
\text { (W.B.) }\end{array}$ & Dioecious,mediumplant,toughrind,mediumfruiting,greencolourfruits \\
\hline DRMC-25 & $\begin{array}{l}\text { Kalayani } \\
\text { W.B.) }\end{array}$ & $\begin{array}{l}\text { Dioecious, vigorousplant,softrind,moreseed,mediumfruiting, lightyellow } \\
\text { greenfruits }\end{array}$ \\
\hline $\begin{array}{l}\text { Spinegourd(Momordica } \\
\text { dioica) }\end{array}$ & & $\begin{array}{l}\text { Dioecious, rootsdevelopssmalltubers,smallleaves, flowersaresmallyellow } \\
\text { colourandopenintheevening,nocirculardotsatthebaseofpetal,roundto } \\
\text { ovalfruits, fruitsaredarkgreentoyellowincolour }\end{array}$ \\
\hline VRMD-1 & Varanasi & Dioecious,mediumviny,morefruiting \\
\hline RSR/DR-1 & Mirzapur & Dioecious,longviny,mediumfruiting \\
\hline RSR/DR-2 & Mirzapur & Dioecious, mediumviny,morefruiting \\
\hline RSR/DR-3 & Sonbhadra & Dioecious,mediumviny,less fruiting \\
\hline
\end{tabular}




\begin{tabular}{|l|l|l|}
\hline VRMD-20-3 & Satana & Dioecious,smallviny, mediumfruiting \\
\hline VRMD-22-5 & Satana & Dioecious,mediumviny,less fruiting \\
\hline VRMD-4 & Patana & Dioecious,smallviny,morefruiting \\
\hline $\begin{array}{l}\text { Balsampear(Momordica } \\
\text { balsamina) }\end{array}$ & $\begin{array}{l}\text { Monoecious,trailingvine withverythinstemsandtendrils, Theleavesare heart- } \\
\text { shapedand3-6cmindiameter,cutinto3-4lobes,Flowersareborne } \\
\text { singlyintheleafaxils,fruitsaresmallhavingridgesthroughout } \\
\text { surfaceandintheendelongationtake place,splitopen,revealingorange } \\
\text { fleshandbrightredplacentatowhichthe seedsareattached,Seedsare } \\
\text { small,lightyellowandoval. }\end{array}$ \\
\hline DRBS-1 & Ranchi & $\begin{array}{l}\text { Monoecious,thinandmediumspreadvines,boldsmallfruits withprominent } \\
\text { distalend. }\end{array}$ \\
\hline $\begin{array}{l}\text { DRBS-2 } \\
\text { (Momordica } \\
\text { cochinchinensis })\end{array}$ & ANGARU & $\begin{array}{l}\text { Monoecious,thinandvigorousvines,mediumprominentdistalend,verysmall } \\
\text { fruits }\end{array}$ \\
\hline DRMC11 & $\begin{array}{l}\text { Bhagalpur } \\
\text { (Bihar) }\end{array}$ & $\begin{array}{l}\text { Dioecious,Dioecious,vigorousplant,softrind,moreseed,mediumfruiting, } \\
\text { lightyellowgreenfruits }\end{array}$ \\
\hline
\end{tabular}

Fieldevaluationanddatacollection

Forfieldevaluation, theaccessionswereplantedfollowingtherecommended fertilizerdoseandculturalpracticestoraiseagood chosenwithineachaccessionfordatarecording, whichwasdoneforfifteen quantitativetraitsi.e.daysoffloweranthesis, ediblematuration, branchesperplant, fruitlength,fruit/plant,fruitweight,seed/fruit,yield/plant,petiolelength nodetofirstfemale floweremergenceandinter-nodallength.

crop.Fiveplantswererandomly

\section{ExtractionofgenomicDNAandquantification}

TotalgenomicDNAwasextractedfrom thefreshyoung leavescollectedfrom fieldintheweehoursoftheday followingthe proceduredescribedbyDoyleandDoyle(1990)andmodificationsofMaguireetal. (1994).TheDNA solutionwaspurifiedandquantityoftheDNAwasestimatedbyrecordingODofspectrophotometer(GeneSpaceIII,Hitachi,Japan)at260nm, andqualitywascheckedon0.8\%agarosegel.

Polymerasechainreaction(PCR)assay andelectrophoresis Outof120randomdecamerprimersfromOperonTechnologies, selectedforRAPDanalysis. Thirty-fivecommerciallyavailableuniversalISSRprimersofUBCseriesand16SSR primers reportedinanearlierstudy(Wangetal.2010)wereusedinthePCR assay.PCRreactionswereperformed in96wellplatesusingBio-radthermo-cyclersystemwiththesuitableamplification randomdecamerprimers(OperonTechnologies,USA).Each25 $\mu$ lreactionmixturecontainedabout50ngof genomicDNA, $0.2 \mu$ Mprimer, $100 \mu$ MeachofdATP,dGTP,dCTPanddTTP, $25 \mathrm{mMMgCl2and0.5UofTaqDNApolymerase}$ alongwithsuitable10xbuffer(10mMTris-Cl,pH8.3,50mMKCl,0.001\%gelatin).The reactionwascarriedoutat $94^{\circ} \mathrm{C}$ for4minaspre-denaturationstep, thentherastion reded 40 timesat $94^{\circ} \mathrm{Cfor}$ $1 \mathrm{~min}, 33^{\circ} \mathrm{C}$ for 1 minandextensionat $72^{\circ} \mathrm{C}$ for $1 \mathrm{~min}$. Additionally, afinalcycleallowedextensionat $72^{\circ} \mathrm{Cfor} 10 \quad \mathrm{~min}$. ForISSRandSSRassay,annealingtemperaturewasstandardizedbeforeperformingtheassayoncompleteset ofaccessions.Carewastaken toensurethatasetofallaccession tobecomparedwereprocessedinthesame machine.

ThePCRamplifiedproductsfromRAPDandISSRassaywereloadedin1.5\%agarosegelandseparated byelectrophoresis with1XTAEbufferat65Vfor1hrand30minutes. Thebandsizeswereestimatedby comparingwith bandsof1KbDNAladder (MBIFermentas), whichwas the amplifiedproductsina separatelaneonthesamegel.ForproductsfromSSRassay, thefragmentswereelectrophoresed through2.5\% Metaphoragarose(FMCBioproducts,USA)gelataconstantvoltageof90volts for5hours.Fragmentlengths were determinedwiththehelpof50bpladder(MBIFermentas,Germany)loadedseparately inthegelalongwiththe samples.Thegelswerestainedwithethidiumbromide $(0.5 \mathrm{mg} / \mathrm{ml})$ andvisualizedinagel-documentedsystem (AlfaImager2200,Alfa-InnotechCorporation,California).ThePCRamplificationwasrepeatedtwotimesto ensurethattheamplificationobtainedwiththeprimersisconsistentandreproducible. 


\title{
Dataanalysis \\ Morphologicalanalysis
}

Themean valueofthe datawasconsideredforcalculatingthegeneticdistanceamongthe ofNTSYS-pcwasusedtoachievethesame.TheNeiandLigeneticdistancematrixwas forgeneratingUnweighted Pair Group Method with (UPGMA)baseddendrogramusingSAHNclustermoduleofNTSYS-pc.

accessions. DISTmodule usedasinput file Arithmetic Mean

\begin{abstract}
Molecularanalysis
PCRamplifiedfragmentsofthe 24accessionwere scoredas present(1)orabsent(0) ofbands. Onlyclear,unambiguous ampliconsrangingfrom300bpto3000bpwerescored.Uniquepresenceorabsenceof singleorgroupofbandswasusedforidentificationofspecies.Resolvingpower content(PIC)ofprimerswerecalculatedtotesttheefficiencyoftheseparameters (Rp)andpolymorphicinformation

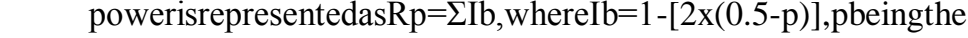
proportionofcultivars containingthebands(PrevostandWilkinson 1999).PIC=1-(p2-q2), where p2isproportionof accessionshavinganampliconandq2isproportionofaccessionsnothavingtheamplicon(Rainaetal.2001).PIC valueofa primerwascalculatedbyaveragingthePICvaluesofallpolymorphicfragmentsgeneratedbytheprimer.
\end{abstract}

Thegeneticassociations betweenaccessionswereevaluatedbycalculatingthemodifiedNeiandLi's coefficientNL $=1$ [2N11/(2N11+N10+N01)](NeiandLi,1979)forpairwisecomparisonsbasedontheproportion of sharedbandsproducedbytheprimers, where,N11 isthenumberofbands/allelespresentinbothindividuals,N10is thenumberofbands/alleles presentonlyinindividualAbutabsent inBandN01isthenumber ofbands/alleles presentonlyinindividualBbutabsentinA. Binarydatabasedonpresence(1)orabsence(0)ofbandswas analyzedbypairwisecomparison usingJaccard'scoefficient(Jaccard1908). Thesimilaritymatrixthusobtained wassubjectedtoclusteranalysisbyUPGMAanddendrogramwasgeneratedtostudytherelatednessofthe cultivars. Therobustnessofthenodesofthedendrogramwastestedbybootstrapanalysisusing 1000resamplings. TheseanalyseswerecarriedoutusingFreetreesoftware(Pavliceketal.1999).UPGMAdendrogramwasdrawn usingTreeview(Page1996).

\section{Results}

Morphologicalevaluationbasedonquantitativetraits

Themeanvalueof15quantitative Momordica (Supplementary surprising. Thedaysofflower (32.5and 40.5 respectively) is Table S1)and highmorphologicaldiversityamongtheseaccessionsisnot anthesisandediblematurityismaximum(79.5and101.2)inDRMC-25andminimum 5, whereas, itisminimum (4.15)inVRMD-20-3. ThegenotypeDRMC22showmaximumfruitweightandseedperfruit(126.35and24, respectively), whileitisminimuminVRMD-22-5 andDRBS-1(10.87and9)andgenotypeRSR/DR-3 showhigh seednumberofper fruit.ThemaximumyieldperplantisrecordedinRCSG-1(2.255kg)andminimuminVRMD-1 (0.365kgs).ThegenotypeVRMD-20-3showhighest fruitsperplant(41.25)andisminimum(10.25)inDRBS-1.

\section{Clusteranalysisbasedonquantitativetraits}

Dendrogramobtainedfromclusteranalysisofthedistancematrixfromthemeanvalueofthe15quantitativetraits

forthe24genotypes (Fig. 1)groupalltheaccessions intotwomajorclustersandseparatesatadistanceof29.00. Perusalofthedendrogramrevealstwomajorclusters. AlltheaccessionsofM.charantia(Gy-323,Gy-333,DRAR1, MC84,PDM,VRBT1,DVBTG7andDVBTG5);M.cochinchinensis(VRMD1,VRMD203,RSRDR3,RSRDR2, RSRDR1, VRMD225 andVRMD4) andM.balsamina(DRBS1andDRBS2)constituted distinctgroupswithinthe majorcluster.The secondmajorclusterconsistedofall accessionofM.dioica.However,RCSG-7wasdistinctfrom othersix genotypesand formeda distinctsub-cluster. 


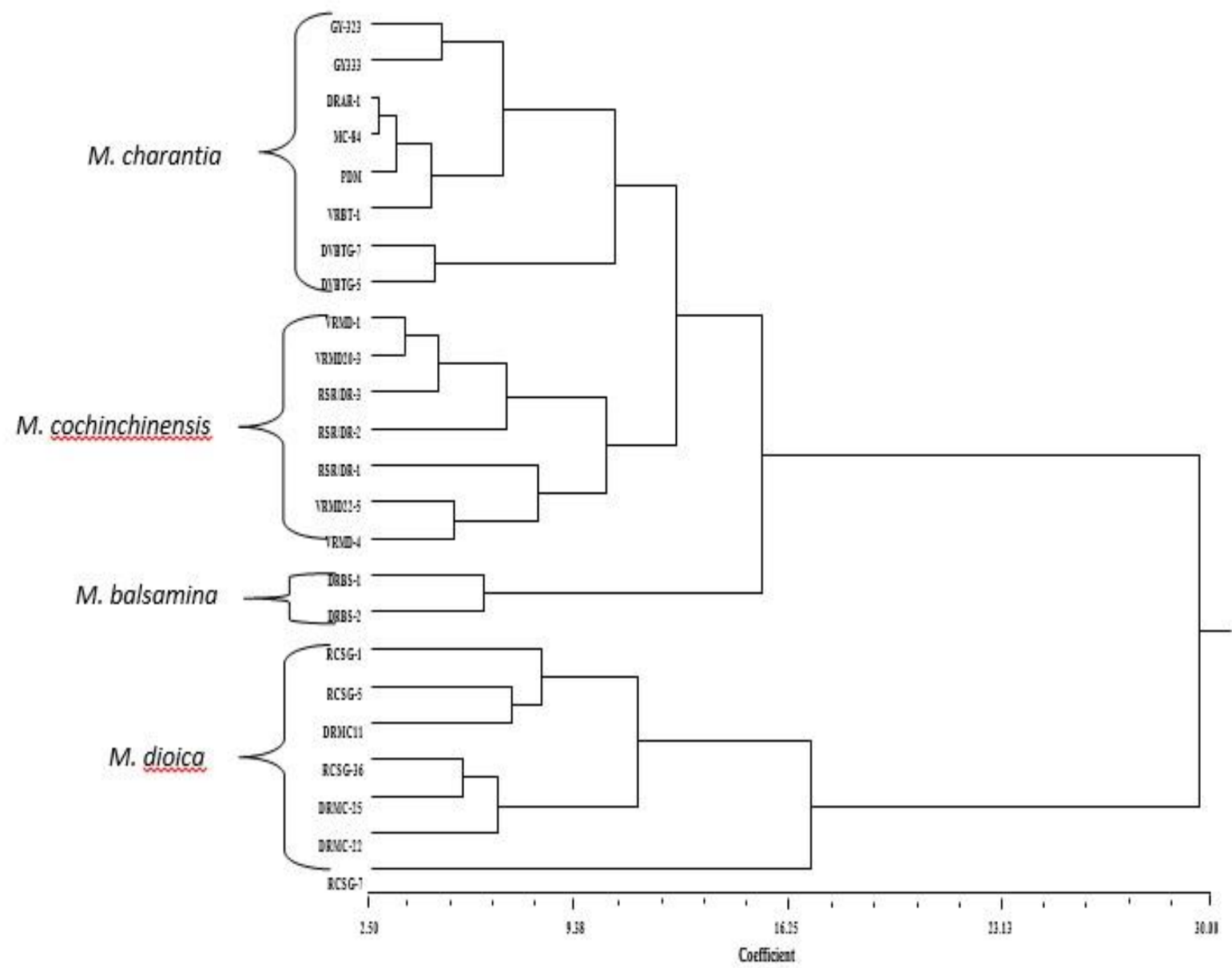

Figure 1: UPGMA dendrogramof Momordicaaccessionsgeneratedfrommeanvaluesof15quantitativetraitsused forclusteranalysisbasedonNei and Ligeneticdistance matrix.

\section{Molecularvariationandspeciesrelationships}

ThesequenceoftheRAPD,ISSRandSSRprimersusedforthemoleculargeneticfingerprinting Momordicaspeciesandthebasicbandsproducedbyeachprimer,numberofpolymorphic polymorphismproducedbyeachprimerarepresentedinsupplementary Table

ofthefour S4,respectively.Thebasicbandstatisticsof obtainedfrom thevarioussetofprimersindifferent combinationisalsogiven intable 2.Theprobabilityofchance identityoftwoaccessionswas $5.66 \times 10$ 247indicatingthehighefficiencyofmarkersindifferentiatingtheaccessions.

Geneticdiversitywithinandamongspeciesassessedusing Nei'sgeneticdiversityandShannoninformationindexis givenintable3andsupplementary Table

S5. Thishighlevelofpolymorphismcaneasilybeattributedtotheaccessionsrepresenting diversespeciesinthestudy.Theresultsarenotincongruencewithearlierstudies(Deyetal.2006andBeheraetal. 2008)duetonon-inclusion ofaccessionsrepresenting suchdiversespecies.Rasuletal.(2007)includedone accessionofM.dioicaasanout-groupto studydiversityinM.charantiausingRAPDmarkers. 
Table2: AnalysisofbandingpatterngeneratedbytheRAPD,SSRandISSRmarkerandcomparativeanalysisof differentmarkersystems.

\begin{tabular}{|l|l|l|l|l|l|l|l|}
\hline \multicolumn{1}{|c|}{ Component } & RAPD & \multicolumn{1}{|c|}{ SSR } & \multicolumn{1}{|c|}{ ISSR } & $\begin{array}{c}\text { RAPD } \\
\text { +SSR }\end{array}$ & $\begin{array}{c}\text { RAPD } \\
\text { +ISSR }\end{array}$ & \multicolumn{1}{|c|}{$\begin{array}{c}\text { SSR+ } \\
\text { ISSR }\end{array}$} & $\begin{array}{c}\text { RAPD+ } \\
\text { SR+ISSR }\end{array}$ \\
\hline No.ofprimers & 50 & 16 & 35 & 66 & 85 & 51 & 101 \\
\hline No.ofmarkers & 264 & 63 & 273 & 327 & 537 & 336 & 600 \\
\hline No.ofmarkersperprimer & 5.28 & 3.93 & 7.8 & 9.21 & 13.08 & 11.73 & 16.49 \\
\hline Polymorphicmarkers & 255 & 61 & 270 & 316 & 525 & 331 & 586 \\
\hline $\begin{array}{l}\text { Polymorphicmarkersper } \\
\text { primer }\end{array}$ & 5.10 & 3.81 & 7.71 & 4.80 & 6.18 & 6.50 & 5.80 \\
\hline Polymorphism(\%) & 96.6 & 95.62 & 99.03 & 96.11 & 97.81 & 97.32 & 97.08 \\
\hline Assayefficiencyindex & 5.1 & 3.81 & 7.71 & 8.91 & 12.81 & 11.52 & 16.62 \\
\hline Markerindex & 72.93 & 75.73 & 78.70 & 148.66 & 151.63 & 154.43 & 227.36 \\
\hline Averageresolvingpower & 3.244 & 1.933 & 4.957 & 5.177 & 8.201 & 6.89 & 10.134 \\
\hline AveragePIC & 0.755 & 0.792 & 0.794 & 1.547 & 1.549 & 1.586 & 2.341 \\
\hline $\begin{array}{l}\text { CorrelationbetweenRP and } \\
\text { PIC }\end{array}$ & 0.053 & 0.521 & 0.083 & 0.574 & 0.136 & 0.604 & 0.657 \\
\hline SizeofPCRproducts(bp) & $300-$ & $150-500$ & $300-$ & $150-$ & $300-$ & $150-$ & $150-3000$ \\
\hline
\end{tabular}

Table 3:Nei'sgeneticdiversityandShannon'sinformationindexvalueswithinthespecies.

\begin{tabular}{|l|c|l|l|l|l|l|l|l|}
\hline $\begin{array}{l}\text { Momordicasp. } \\
\text { Name }\end{array}$ & \multirow{2}{*}{$\begin{array}{l}\text { Sample } \\
\text { size }\end{array}$} & $\begin{array}{l}\text { Numberof } \\
\text { polymorphic } \\
\text { loci(\%) }\end{array}$ & $\begin{array}{l}\text { Nei'sgenetic } \\
\text { diversity }\end{array}$ & Mean & St.Dev & $\begin{array}{l}\text { Shannon's } \\
\text { informationindex }\end{array}$ & \multicolumn{2}{|c|}{ HsandHt* } \\
\hline M.charantia & 8 & $153(25.37)$ & 0.0999 & 0.1838 & 0.1454 & 0.2616 & 0.0999 & 0.0338 \\
\hline M.cochinchinensis & 7 & $274(45.44)$ & 0.1572 & 0.1959 & 0.2357 & 0.2811 & 0.1572 & 0.0384 \\
\hline M.dioica & 7 & $235(38.97)$ & 0.1448 & 0.1999 & 0.2136 & 0.2854 & 0.1448 & 0.0400 \\
\hline M.balsamina & 2 & $108(17.91)$ & 0.0742 & 0.1590 & 0.1803 & 0.2321 & 0.0742 & 0.0253 \\
\hline Momordicasp. & 24 & $589(97.68)$ & 0.3136 & 0.1274 & 0.4808 & 0.1622 & 0.3511 & 0.0186 \\
\hline
\end{tabular}

Htvalueisforcompletesetof24accessionsdepictingthediversityinthe setofaccessionsincludedinthestudy.

Theaboveresultsclearly distinguish bothmonoecious speciesfromthedioeciousonesandthespecies withinboth groups.Uniquemarkersdistinguishingboth thegroupsandsomespecies-specificmarkerscouldalsobe identifiedthroughthestudy(supplementary Table S6).Seventeenmarkerscouldbeidentified differentiating monoecious and dioecious species, while85ampliconsspecifictooneofthefourMomordicaspecieswereidentified.Theseresults highlightthepotentialofmolecularmarkersinidentifying accessionsatspecieslevelandaccessingthegenetic diversityofaccessionsbothwithinandacrossspecies.

\section{Discussion}

Morphologicalevaluationbasedonquantitativetraits

SeveralspeciesofgenusMomordicaoccurinIndia.AmongthemM.charantia,M.dioica,M.cochinchinensisand M.balsaminaaredistributedacrossvariousagro-climaticzonesofIndia,Theaccessionsincludedinthis studywere collectedfromtheir respective zones, representingfourspecies:twoeachofmonoecious and dioeciousnature(Table1).

\section{Clusteranalysisbasedonquantitativetraits}

Thedendrogramobtainedfromclusteranalysisofthedistinctmatrixformedthemeanvalueoffifteenquantitative traitsclearlyexhibitsdistinction amongaccession offourMomordicaspeciesrepresentedinthestudy.Further, $M$. dioicaformsdistinctclusters whilethe other three speciesgroupin separatecluster formingdifferentgroupsofeach specieswithinthecluster.This couldprobablebe becausethetraits evaluatedduringthestudydidnotinvolvethose featuresof floralbiology. This clusteringpatternof speciescould not establishanycorrelationbetweenthe clusteringpatternswiththegeographicaldistributionoftheaccessions.Deyetal.(2006)alsocouldnotestablishany suchcorrelationforM.charantiaaccessions.However,non-congruence oftheresultswithpreviousstudy(Deyet al.,2006)couldbeduetoinclusionofmorenumberofspeciesinthestudyandrecordingdifferentquantitativetraits foranalysis. TheresultsareinpartialcongruencewithBharathietal.(2012)onrelatedness ofspeciesdemonstrated throughcrossability. 
Themorphologicaltraitsrecordedinthisstudy,couldefficientlyclusteralltheaccessionsrepresenting a species, thesetraitscanbehighlyeffectiveindiversityanalysis ofasetofgermplasmandindevelopingcoresetof thegermplasmrepresentingmaximumdiversityacrossthespecies.Kumaretal.(2010)haveadvocated useof strategyofidentifyinghighlyvariabledescriptorsforcapturingmaximumgeneticdiversityinthecoreset.

AmongM.charantiaaccessions,twogynoecious lines(Gy-323,Gy-333)recordedearlydaystoflower anthesisanddaystoediblematurityindicatingearlinessandearlyharvest.Theselinesalsoexhibitmaximumfruit perplant,fruitweightandyieldperplantindicatingtheirpotentialtodevelophighyieldinggynoeciousvarietyin futurebreedingprogramme (Rametal.,2002,Deyetal., 2006,Beheraetal., 2008 and Shukla et al., 2015).However, plants respondandadaptdifferentlytoselectionpressuresimposedbydistinctagroclimaticzones(Singhetal., 1998). Theseresultshighlightthepotentialofquantitativetraitsindistinguishingaccessionatspecieslevel.Rasuletal., 2007alsoreportedsimilardistinctionsofspeciesusingRAPDmarkers.

\section{Molecularvariationandspeciesrelationships}

Thegeneticrelationshipamongthefourspeciesis

assessedbasedonJaccard'ssimilaritycoefficientfromthe pooleddatafrom differentmarkersystemsanddendrogramisgeneratedusingun-weighed pairgroupmethodwith arithmeticaverages(UPGMA). Therobustness ofnodesinthedendrogramisassessedbybootstrapanalysisby analyzingthedatawith1000replications(Fig.2)usingFreetreeandTreeviewsoftwares. Theperusalofthe dendrogramshowstwodistinctclustersofmonoeciousanddiociousspecieswithbootstrapvalueof100atnode. Thefirstmajorclusterincludestheaccessionfrom M.cochinchinensiswithM.dioicaspecieswhilethesecond consistsofaccessionfrom M.charantiawithM.balsamina. Theresultisalsosupportsthediversity plastids,mitochondriaandnuclearDNA, where M.dioicaandM.cochinchinensisarenestedinaclade(Schaefer

analysisof andRenner,2010). Thebootstrapvaluesatthenodesdifferentiating speciesarehightoalevelof100for $M$. cochinchinenesisand M.dioica,M.charantiaandM.balsamina.Theclusteringpatternclearlydivides4groups, eachrepresenting asinglespecies includedinthisstudy.Thebootstrapvaluesrangesfrom cochinchinensis;22to100forMdioicaand40to100forMcharantiarepresentinghighlevelofdiversityin accessionofthesespecies. The twoaccessionofM.balsaminaincludedin thisstudywerehighlydiversefromeach other withbootstrapvalueof100 for nodeconnectingthem.

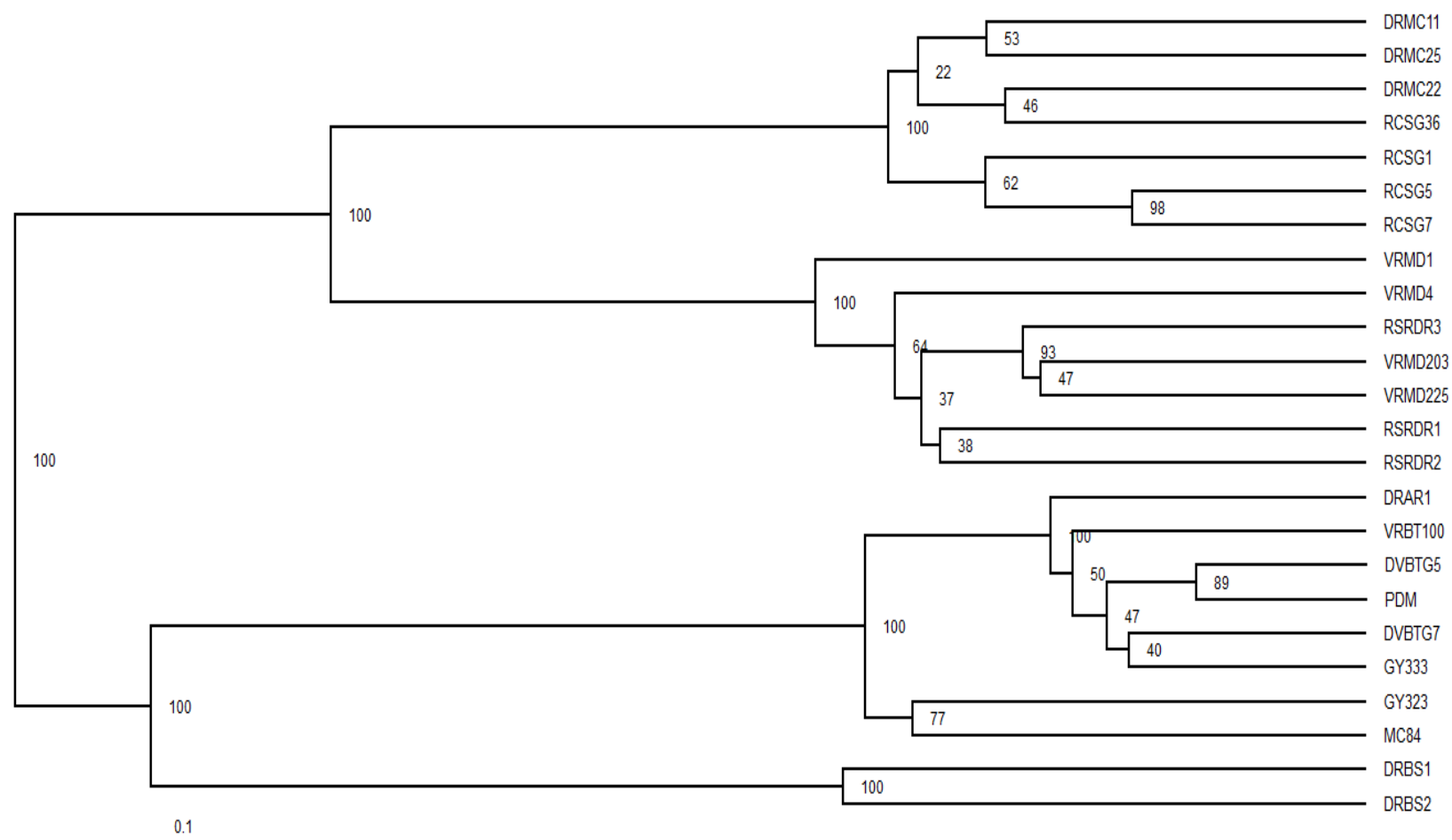

Figure2:UPGMAdendrogramofMomordicaaccessionswithbootstarpvaluesfromJaccard'ssimilarity coefficientobtainedfrommoleculardataanalysis. 
Theperusalofdendrogramgeneratedfromthemoleculardatathrough1000replicationforbootstrap revealsresolution offourdistinctclusterseachrepresenting aspecieswithintermediate tohighbootstrapvaluesat thenodesrevealinghighgeneticheterogeneity amongtheaccessionsofthespecies.Similarresultsofhighgenetic diversitywithinspecieshavebeenreported(Deyetal.,2006;Rasuletal.,2007;Beheraetal.,2008;Dalamuetal., 2012andJietal.,2012).

Basedonmoleculardata,101primersgenerated600 scorableamplicons across fourspecies, ofwhich586polymorphic ampliconsexhibiting $97.08 \%$ polymorphismamongthesamples.This high level of polymorphismcan beattributedtotheaccessionsrepresenting

diversespeciesinthestudy.Theresultsarenotincongruencewithearlierstudies(Deyetal.,2006;Beheraetal., 2008andDalamuetal.,2012)duetonon-inclusionofaccessionsrepresentingsuchdiversespecies.Rasuletal.

(2007)includedoneaccessionofM.dioicaasanout-groupto studydiversityinM.charantiausingRAPDmarkers.

Seventeen markerscouldbeidentifieddifferentiating monoecious and dioeciousspecies, while85 amplicons specifictooneofthefourMomordicaspecieswereidentified.Theseresultshighlightthepotential of molecularmarkersinidentifyingaccessions atspecieslevelandaccessing thegeneticdiversityofaccessions both withinandacrossspecies. Theperusalofdendrogramgeneratedfromthemoleculardatathrough1000replication

forbootstraprevealsresolutionoffourdistinctclusterseachrepresenting aspecieswithintermediatetohigh bootstrapvaluesatthenodesrevealinghighgeneticheterogeneity amongtheaccessions ofthespecies. Similar resultsofhighgeneticdiversitywithin specieshave been reported(Deyetal.,2006; Rasuletal., 2007; Beheraetal., 2008andJietal.,2012).

Inthepresentstudy,morphological diversity basedonquantitativetraitsandmoleculardiversityshowedsome correspondenceintheclusteringpattern ofaccessionsrepresentingaspecies. Allthespeciescouldbedistinguished clearly, whilemoretraitsrelatedtofloralbiologyofthespecieswererequiredto distinguish species morphologicallyinthestudy.Thepotentialityofgynoeciouslinesforearlinessandyieldattributesisevidentand

canbeeffectively exploitedinfuturebreedingprograms.Thoughaccessions ofsamespeciesgroupedtogetherin bothmorphological andmolecularanalysis,molecularmarkersweremoreefficientthanmorphologicalparameters innotonlydifferentiatingtheaccessionsat specieslevelbutalsodistinguishingmonoeciousanddioeciousspecies.

\section{Supplementary Table}

Supplementary Table S1:MorphologicalperformanceofMomordicasp. genotypesmeasuredfor15quantitativetraits

\begin{tabular}{|c|c|c|c|c|c|c|c|c|c|c|c|c|c|c|c|}
\hline $\begin{array}{l}\text { Acces } \\
\text { sions }\end{array}$ & $\begin{array}{l}\text { Days } \\
\text { to } \\
\text { flow } \\
\text { er } \\
\text { anth } \\
\text { esis } \\
\text { (Day } \\
\text { s) }\end{array}$ & $\begin{array}{l}\text { Edibl } \\
\text { e } \\
\text { matu } \\
\text { ratio } \\
\text { n }\end{array}$ & $\begin{array}{l}\text { Bran } \\
\text { ches } \\
\text { /Pla } \\
\text { nt }\end{array}$ & $\begin{array}{l}\text { Pla } \\
\text { nt } \\
\text { hei } \\
\text { ght } \\
\text { (c } \\
\text { m) }\end{array}$ & $\begin{array}{l}\text { Frui } \\
t \\
\text { dia } \\
\text { mete } \\
\text { r } \\
\text { (cm) }\end{array}$ & $\begin{array}{l}\text { Fr } \\
\text { uit } \\
\text { len } \\
\text { gth } \\
\text { (c } \\
\text { m) }\end{array}$ & $\begin{array}{l}\text { Fr } \\
\text { uit } \\
\text { /PI } \\
\text { ant }\end{array}$ & $\begin{array}{l}\text { Fr } \\
\text { uit } \\
\text { wei } \\
\text { ght } \\
\text { (g) }\end{array}$ & $\begin{array}{l}\text { Se } \\
\text { ed } \\
\text { s } \\
/ \text { Fr } \\
\text { uit }\end{array}$ & $\begin{array}{l}\text { Petiol } \\
\text { e } \\
\text { lengh } \\
t(\mathrm{~cm})\end{array}$ & $\begin{array}{l}\text { Ped } \\
\text { icle } \\
\text { len } \\
\text { gth } \\
\text { (cm } \\
\text { ) }\end{array}$ & $\begin{array}{l}\text { Node } \\
\text { to1st } \\
\text { male } \\
\text { flow } \\
\text { er }\end{array}$ & $\begin{array}{l}\text { Node } \\
\text { to1stf } \\
\text { emale } \\
\text { flowe } \\
r\end{array}$ & $\begin{array}{l}\text { Int } \\
\text { er } \\
\text { no } \\
\text { dal } \\
\text { len } \\
\text { gth } \\
\text { (c } \\
\text { m) }\end{array}$ & $\begin{array}{l}\text { Yi } \\
\text { eld } \\
\text { /PI } \\
\text { ant } \\
\text { (kg } \\
\text { ) }\end{array}$ \\
\hline \multicolumn{16}{|c|}{ Mcharantia } \\
\hline $\begin{array}{l}\text { GY- } \\
323\end{array}$ & 34.2 & 44.2 & 9 & $\begin{array}{l}95 . \\
3\end{array}$ & 2.96 & $\begin{array}{l}15 . \\
29\end{array}$ & $\begin{array}{l}20 . \\
75\end{array}$ & $\begin{array}{l}44 . \\
5\end{array}$ & 15 & 5.6 & 9.8 & 7.2 & 11.3 & $\begin{array}{l}10 . \\
2\end{array}$ & $\begin{array}{l}1.9 \\
8\end{array}$ \\
\hline $\begin{array}{l}\text { GY33 } \\
3\end{array}$ & 32.5 & 40.5 & 11 & $\begin{array}{l}11 \\
0.5\end{array}$ & 2.65 & $\begin{array}{l}18 . \\
75\end{array}$ & $\begin{array}{l}16 . \\
25\end{array}$ & $\begin{array}{l}38 . \\
35\end{array}$ & 12 & 6.3 & $\begin{array}{l}10 . \\
2\end{array}$ & 8.3 & 12.1 & 9.5 & $\begin{array}{l}1.8 \\
5\end{array}$ \\
\hline $\begin{array}{l}\text { DRA } \\
\text { R-1 }\end{array}$ & 47.3 & 52.6 & 7 & $\begin{array}{l}11 \\
5.4\end{array}$ & 3.33 & $\begin{array}{l}16 . \\
66\end{array}$ & $\begin{array}{l}14 . \\
47\end{array}$ & $\begin{array}{l}36 . \\
56\end{array}$ & 16 & 5.4 & 9.5 & 9.4 & 13.2 & $\begin{array}{l}11 . \\
2\end{array}$ & $\begin{array}{l}1.2 \\
6\end{array}$ \\
\hline $\begin{array}{l}\text { VRB } \\
\text { T- } \\
100\end{array}$ & 39.4 & 49.8 & 13 & $\begin{array}{l}12 \\
5.2\end{array}$ & 3.47 & $\begin{array}{l}15 . \\
33\end{array}$ & $\begin{array}{l}13 . \\
65\end{array}$ & $\begin{array}{l}34 . \\
06\end{array}$ & 18 & 5.1 & 8.7 & 7.6 & 10.5 & 8.9 & $\begin{array}{l}1.4 \\
3\end{array}$ \\
\hline $\begin{array}{l}\text { MC- } \\
84 \\
\end{array}$ & 41.7 & 52.2 & 10 & $\begin{array}{l}11 \\
3.4\end{array}$ & 3.12 & $\begin{array}{l}10 . \\
25\end{array}$ & $\begin{array}{l}15 . \\
23\end{array}$ & $\begin{array}{l}35 . \\
55\end{array}$ & 14 & 3.2 & 9.1 & 8.8 & 12.5 & 7.9 & $\begin{array}{l}1.6 \\
6\end{array}$ \\
\hline $\begin{array}{l}\text { DVB } \\
\text { TG- } \\
7\end{array}$ & 45.5 & 50.5 & 9 & $\begin{array}{l}14 \\
5.2\end{array}$ & 3.26 & $\begin{array}{l}12 . \\
6\end{array}$ & $\begin{array}{l}11 . \\
82\end{array}$ & $\begin{array}{l}40 . \\
25\end{array}$ & 16 & 4.6 & $\begin{array}{l}12 . \\
2\end{array}$ & 5.6 & 9.8 & 9.9 & $\begin{array}{l}1.8 \\
2\end{array}$ \\
\hline
\end{tabular}




\begin{tabular}{|c|c|c|c|c|c|c|c|c|c|c|c|c|c|c|c|}
\hline $\begin{array}{l}\text { DVB } \\
\text { TG- } \\
5\end{array}$ & 42.3 & 54.1 & 15 & $\begin{array}{l}15 \\
3.6\end{array}$ & 2.62 & $\begin{array}{l}18 . \\
98\end{array}$ & $\begin{array}{l}12 . \\
65\end{array}$ & $\begin{array}{l}30 . \\
28\end{array}$ & 12 & 5.5 & $\begin{array}{l}10 . \\
5\end{array}$ & 7.8 & 10.2 & $\begin{array}{l}11 . \\
3\end{array}$ & $\begin{array}{l}1.3 \\
4\end{array}$ \\
\hline PDM & 49.1 & 58.6 & 6 & $\begin{array}{l}11 \\
4.5\end{array}$ & 4.11 & $\begin{array}{l}9.5 \\
5\end{array}$ & $\begin{array}{l}10 . \\
52\end{array}$ & $\begin{array}{l}35 . \\
32\end{array}$ & 13 & 6.2 & $\begin{array}{l}13 . \\
9\end{array}$ & 9.4 & 13.7 & $\begin{array}{l}10 . \\
5\end{array}$ & $\begin{array}{l}0.9 \\
8\end{array}$ \\
\hline \multicolumn{16}{|c|}{ M.cochinchinensis } \\
\hline $\begin{array}{l}\text { RCS } \\
\text { G-1 }\end{array}$ & 58.5 & 73.6 & 20 & $\begin{array}{l}19 \\
0.5\end{array}$ & 4.35 & $\begin{array}{l}7.5 \\
2\end{array}$ & $\begin{array}{l}24 . \\
55\end{array}$ & $\begin{array}{l}112 \\
.5 \\
5\end{array}$ & 20 & 4.9 & $\begin{array}{l}15 . \\
6\end{array}$ & 18.3 & 25.4 & $\begin{array}{l}15 . \\
5\end{array}$ & $\begin{array}{l}2.2 \\
5\end{array}$ \\
\hline $\begin{array}{l}\text { RCS } \\
\text { G-7 }\end{array}$ & 55.5 & 70.2 & 18 & $\begin{array}{l}21 \\
0.5\end{array}$ & 3.98 & $\begin{array}{l}6.5 \\
3\end{array}$ & $\begin{array}{l}36 . \\
58\end{array}$ & $\begin{array}{l}89 . \\
54\end{array}$ & 19 & 3.7 & $\begin{array}{l}13 . \\
2\end{array}$ & 20.1 & 23.5 & $\begin{array}{l}12 . \\
3\end{array}$ & $\begin{array}{l}1.9 \\
8\end{array}$ \\
\hline $\begin{array}{l}\text { RCS } \\
\text { G-5 }\end{array}$ & 63.2 & 81.5 & 19 & $\begin{array}{l}16 \\
5.3\end{array}$ & 4.85 & $\begin{array}{l}5.1 \\
4 \\
\end{array}$ & $\begin{array}{l}21 . \\
35\end{array}$ & $\begin{array}{l}96 . \\
25\end{array}$ & 18 & 5.6 & $\begin{array}{l}14 . \\
6\end{array}$ & 21.4 & 27.4 & $\begin{array}{l}15 . \\
7\end{array}$ & $\begin{array}{l}1.4 \\
5\end{array}$ \\
\hline $\begin{array}{l}\text { RCS } \\
\text { G-36 }\end{array}$ & 75.8 & 90.5 & 19 & $\begin{array}{l}14 \\
4.5\end{array}$ & 4.65 & $\begin{array}{l}4.9 \\
5\end{array}$ & $\begin{array}{l}18 . \\
45\end{array}$ & $\begin{array}{l}114 \\
.5 \\
6\end{array}$ & 16 & 5.9 & $\begin{array}{l}15 . \\
8\end{array}$ & 19.8 & 26.5 & $\begin{array}{l}11 . \\
2\end{array}$ & $\begin{array}{l}2.1 \\
0\end{array}$ \\
\hline $\begin{array}{l}\text { DRM } \\
\text { C- } \\
22\end{array}$ & 68.9 & 88.6 & 14 & $\begin{array}{l}13 \\
5.6\end{array}$ & 3.25 & $\begin{array}{l}5.1 \\
1\end{array}$ & $\begin{array}{l}16 . \\
87\end{array}$ & $\begin{array}{l}126 \\
.3 \\
5\end{array}$ & 24 & 4.5 & $\begin{array}{l}15 . \\
6\end{array}$ & 14.5 & 18.4 & $\begin{array}{l}14 . \\
7\end{array}$ & $\begin{array}{l}1.5 \\
6\end{array}$ \\
\hline $\begin{array}{l}\text { DRM } \\
\text { C- } \\
25\end{array}$ & 79.5 & 101.2 & 21 & $\begin{array}{l}15 \\
4.8\end{array}$ & 3.88 & $\begin{array}{l}4.6 \\
8\end{array}$ & $\begin{array}{l}21 . \\
54\end{array}$ & $\begin{array}{l}118 \\
.4 \\
7\end{array}$ & 19 & 4.7 & $\begin{array}{l}12 . \\
1\end{array}$ & 11.1 & 18.5 & $\begin{array}{l}13 . \\
6\end{array}$ & $\begin{array}{l}1.3 \\
4\end{array}$ \\
\hline \multicolumn{16}{|c|}{ M.dioica } \\
\hline $\begin{array}{l}\text { VRM } \\
\text { D-1 }\end{array}$ & 50.5 & 65.4 & 11 & $\begin{array}{l}11 \\
9.8\end{array}$ & 2.64 & $\begin{array}{l}4.5 \\
4\end{array}$ & $\begin{array}{l}32 . \\
54\end{array}$ & $\begin{array}{l}12 . \\
54\end{array}$ & 19 & 3.6 & $\begin{array}{l}13 . \\
4\end{array}$ & 12.5 & 14.7 & $\begin{array}{l}11 . \\
2\end{array}$ & $\begin{array}{l}0.3 \\
6\end{array}$ \\
\hline $\begin{array}{l}\text { RSR/ } \\
\text { DR- } \\
1\end{array}$ & 54.6 & 68.5 & 13 & $\begin{array}{l}16 \\
5.6\end{array}$ & 2.95 & $\begin{array}{l}4.6 \\
2\end{array}$ & $\begin{array}{l}39 . \\
21\end{array}$ & $\begin{array}{l}19 . \\
58\end{array}$ & 16 & 3.3 & 8.9 & 10.2 & 13.5 & 8.6 & $\begin{array}{l}0.4 \\
5\end{array}$ \\
\hline $\begin{array}{l}\text { RSR/ } \\
\text { DR- } \\
2\end{array}$ & 60.2 & 75.6 & 9 & $\begin{array}{l}11 \\
4.5\end{array}$ & 2.54 & $\begin{array}{l}4.3 \\
5\end{array}$ & $\begin{array}{l}18 . \\
95\end{array}$ & $\begin{array}{l}16 . \\
54\end{array}$ & 18 & 3.2 & 9.5 & 9.8 & 12.4 & 9.5 & $\begin{array}{l}0.5 \\
4\end{array}$ \\
\hline $\begin{array}{l}\text { RSR/ } \\
\text { DR- } \\
3 \\
\end{array}$ & 41.2 & 62.1 & 14 & $\begin{array}{l}11 \\
0.5\end{array}$ & 2.22 & $\begin{array}{l}4.2 \\
9\end{array}$ & $\begin{array}{l}26 . \\
56\end{array}$ & $\begin{array}{l}14 . \\
25\end{array}$ & 24 & 2.1 & 9.7 & 8.7 & 10.4 & 7.2 & $\begin{array}{l}0.7 \\
4\end{array}$ \\
\hline $\begin{array}{l}\text { VRM } \\
\text { D- } \\
20-3\end{array}$ & 43.2 & 66.3 & 10 & $\begin{array}{l}11 \\
3.2\end{array}$ & 3.24 & $\begin{array}{l}4.1 \\
5\end{array}$ & $\begin{array}{l}41 . \\
25\end{array}$ & $\begin{array}{l}11 . \\
24\end{array}$ & 16 & 3.9 & 6.4 & 12.5 & 16.5 & 6.7 & $\begin{array}{l}0.6 \\
5\end{array}$ \\
\hline $\begin{array}{l}\text { VRM } \\
\text { D- } \\
22-5\end{array}$ & 55.8 & 62.5 & 16 & $\begin{array}{l}14 \\
5.6\end{array}$ & 3.11 & $\begin{array}{l}5.1 \\
1\end{array}$ & $\begin{array}{l}35 . \\
89\end{array}$ & $\begin{array}{l}10 . \\
87\end{array}$ & 13 & 4.5 & 7.8 & 9.6 & 13.2 & 8.5 & $\begin{array}{l}0.5 \\
5\end{array}$ \\
\hline $\begin{array}{l}\text { DRM } \\
\text { C11 }\end{array}$ & 60.5 & 86.2 & 19 & $\begin{array}{l}16 \\
9.5\end{array}$ & 3.26 & $\begin{array}{l}8.5 \\
3\end{array}$ & $\begin{array}{l}19 . \\
55\end{array}$ & $\begin{array}{l}115 \\
.6 \\
4\end{array}$ & 14 & 3.7 & 8.4 & 8.5 & 12.4 & 4.9 & $\begin{array}{l}1.5 \\
6\end{array}$ \\
\hline $\begin{array}{l}\text { VRM } \\
\text { D-4 }\end{array}$ & 55.4 & 68.5 & 12 & $\begin{array}{l}12 \\
9.6 \\
\end{array}$ & 2.98 & $\begin{array}{l}3.6 \\
5 \\
\end{array}$ & $\begin{array}{l}40 . \\
25 \\
\end{array}$ & $\begin{array}{l}18 . \\
11 \\
\end{array}$ & 11 & 4.9 & 7.8 & 9.1 & 11.2 & 5.5 & $\begin{array}{l}0.4 \\
8 \\
\end{array}$ \\
\hline \multicolumn{16}{|c|}{ M.balsamina } \\
\hline $\begin{array}{l}\text { DRB } \\
\text { S-1 }\end{array}$ & 35 & 44.3 & 6 & $\begin{array}{l}65 . \\
8 \\
\end{array}$ & 1.98 & $\begin{array}{l}5.8 \\
2 \\
\end{array}$ & $\begin{array}{l}10 . \\
25\end{array}$ & $\begin{array}{l}28 . \\
51\end{array}$ & 9 & 3.6 & 6.8 & 6.5 & 8.5 & 5.8 & $\begin{array}{l}0.5 \\
8 \\
\end{array}$ \\
\hline $\begin{array}{l}\text { DRB } \\
\text { S-2 }\end{array}$ & 41 & 53.6 & 9 & $\begin{array}{l}85 . \\
3\end{array}$ & 1.85 & $\begin{array}{l}6.8 \\
9\end{array}$ & $\begin{array}{l}13 . \\
65\end{array}$ & $\begin{array}{l}30 . \\
55\end{array}$ & 12 & 2.4 & 9.4 & 5.4 & 6.7 & 3.5 & $\begin{array}{l}0.8 \\
5\end{array}$ \\
\hline Mean & $\begin{array}{l}51.3 \\
1\end{array}$ & 65.05 & $\begin{array}{l}12.9 \\
2\end{array}$ & $\begin{array}{l}13 \\
3.0 \\
7\end{array}$ & 3.22 & $\begin{array}{l}8.4 \\
7\end{array}$ & $\begin{array}{l}22 . \\
20\end{array}$ & $\begin{array}{l}51 . \\
27\end{array}$ & $\begin{array}{l}16 . \\
00\end{array}$ & 4.43 & $\begin{array}{l}10 . \\
78\end{array}$ & 10.92 & 14.68 & $\begin{array}{l}9.7 \\
4\end{array}$ & $\begin{array}{l}1.2 \\
5\end{array}$ \\
\hline S.D. & $\begin{array}{l}12.6 \\
2\end{array}$ & 16.16 & 4.60 & $\begin{array}{l}33 . \\
19\end{array}$ & 0.78 & $\begin{array}{l}5.0 \\
2\end{array}$ & $\begin{array}{l}10 . \\
08\end{array}$ & $\begin{array}{l}40 . \\
48\end{array}$ & $\begin{array}{l}3.8 \\
0\end{array}$ & 1.16 & $\begin{array}{l}2.8 \\
6\end{array}$ & 4.62 & 5.74 & $\begin{array}{l}3.2 \\
5\end{array}$ & $\begin{array}{l}0.6 \\
0\end{array}$ \\
\hline
\end{tabular}


Supplementary Table S2:DetailsofRAPDprimersselectedforthe study

\begin{tabular}{|c|c|c|c|c|c|c|c|}
\hline Primers & Sequence5 to3 & $\operatorname{AT}\left({ }^{\circ} \mathrm{C}\right)$ & $\begin{array}{l}\text { GC } \\
(\%)\end{array}$ & \begin{tabular}{|l|} 
Total \\
bands
\end{tabular} & $\begin{array}{l}\text { Polymorphic } \\
\text { bands(\%) }\end{array}$ & $\mathbf{R p}$ & PIC \\
\hline OPAF-04 & TTGCGGCTGA & 33 & 60 & 8 & $8(100)$ & 5.667 & 0.749 \\
\hline OPAF-06 & CCGCAGTCTG & 33 & 70 & 4 & $3(75)$ & 2.167 & 0.651 \\
\hline OPAF-11 & ACTGGGCCTC & 34 & 70 & 4 & $4(100)$ & 2.917 & 0.613 \\
\hline OPAF-12 & GACGCAGCTT & 33 & 60 & 4 & $4(100)$ & 2.250 & 0.723 \\
\hline OPAF-15 & CACGAACCTC & 33 & 60 & 3 & $3(100)$ & 0.167 & 0.786 \\
\hline OPAG-03 & TGCGGGAGTG & 33 & 70 & 3 & $3(100)$ & 1.833 & 0.865 \\
\hline OPAG-04 & GGAGCGTACT & 33 & 60 & 3 & $3(100)$ & 2.167 & 0.860 \\
\hline OPAG-06 & GGTGGCCAAG & 33 & 70 & 4 & $4(100)$ & 2.833 & 0.849 \\
\hline OPAG-10 & GGTTGGAGAC & 33 & 60 & 7 & $7(100)$ & 3.750 & 0.686 \\
\hline OPAG-11 & TTACGGTGGG & 34 & 60 & 4 & $4(100)$ & 2.417 & 0.749 \\
\hline OPAG-12 & CTCCCAGGGT & 34 & 70 & 4 & $4(100)$ & 3.667 & 0.706 \\
\hline OPAG-15 & CCCACACGCA & 34 & 70 & 4 & $4(100)$ & 2.500 & 0.715 \\
\hline OPC-06 & GAACGGACTC & 33 & 60 & 3 & $3(100)$ & 3.000 & 0.856 \\
\hline OPC-08 & TGGACCGGTG & 34 & 70 & 5 & $5(100)$ & 1.917 & 0.619 \\
\hline OPC-14 & TGCGTGCTTG & 33 & 60 & 3 & $3(100)$ & 5.167 & 0.760 \\
\hline OPC-16 & CACACTCCAG & 33 & 60 & 8 & $8(100)$ & 2.333 & 0.940 \\
\hline OPF-16 & GGAGTACTGG & 33 & 60 & 5 & $5(100)$ & 4.583 & 0.542 \\
\hline OPH-10 & CCTACGTCAG & 34 & 60 & 7 & $7(100)$ & 2.500 & 0.396 \\
\hline OPK-20 & GTGTCGCGAG & 33 & 70 & 5 & $5(100)$ & 4.167 & 0.843 \\
\hline OPL-07 & AGGCGGGAAC & 33 & 70 & 6 & $6(100)$ & 1.167 & 0.985 \\
\hline OPL-08 & AGCAGGTGGA & 33 & 60 & 5 & $4(100)$ & 4.306 & 0.839 \\
\hline OPL-14 & GTGACAGGCT & 33 & 60 & 6 & $6(100)$ & 3.500 & 0.793 \\
\hline OPL-16 & AGGTTGCAGG & 33 & 60 & 7 & $7(100)$ & 4.917 & 0.654 \\
\hline OPL-20 & TGGTGGACCA & 33 & 60 & 10 & $9(90)$ & 5.833 & 0.795 \\
\hline OPM-12 & GGGACGTTGG & 33 & 70 & 7 & $7(100)$ & 4.167 & 0.788 \\
\hline OPM-14 & AGGGTCGTTC & 34 & 60 & 6 & $6(100)$ & 1.083 & 0.237 \\
\hline OPN-08 & ACCTCAGCTC & 33 & 60 & 4 & $4(100)$ & 1.500 & 0.832 \\
\hline OPN-12 & CACAGACACC & 33 & 60 & 4 & $4(100)$ & 3.083 & 0.724 \\
\hline OPN-14 & TCGTGCGGGT & 33 & 70 & 4 & $4(100)$ & 3.250 & 0.870 \\
\hline OPO-06 & CCACGGGAAG & 33 & 70 & 6 & $6(100)$ & 4.167 & 0.792 \\
\hline OPO-10 & TGTGCCCGAA & 33 & 60 & 6 & $6(100)$ & 1.750 & 0.947 \\
\hline OPY-03 & ACAGCCTGCT & 33 & 60 & 4 & $4(100)$ & 5.500 & 0.758 \\
\hline OPY-04 & GGCTGCAATG & 33 & 60 & 7 & $7(100)$ & 2.917 & 0.740 \\
\hline OPY-06 & AAGGCTCACC & 34 & 60 & 4 & $4(100)$ & 5.417 & 0.733 \\
\hline OPY-07 & AGAGCCGTCA & 33 & 60 & 7 & $7(100)$ & 5.250 & 0.770 \\
\hline OPY-08 & AGGCAGAGCA & 33 & 60 & 8 & $8(100)$ & 2.583 & 0.928 \\
\hline OPY-09 & AGCAGCGCAC & 34 & 70 & 5 & $5(100)$ & 2.833 & 0.701 \\
\hline OPY-14 & GGTCGATGTG & 33 & 60 & 4 & $4(100)$ & 2.333 & 0.592 \\
\hline OPY-15 & AGTCGCCCTT & 34 & 60 & 4 & $4(100)$ & 2.917 & 0.639 \\
\hline OPY-16 & GGGCCAATGT & 34 & 60 & 5 & $5(100)$ & 1.917 & 0.634 \\
\hline OPY-17 & GACGTGGTGA & 33 & 60 & 3 & $3(100)$ & 5.500 & 0.717 \\
\hline OPY-18 & GTGGAGTCAG & 33 & 60 & 8 & $8(100)$ & 2.750 & 0.857 \\
\hline OPZ-01 & TCTGTGCCAC & 33 & 60 & 5 & $5(100)$ & 4.250 & 0.767 \\
\hline OPZ-06 & GTGCCGTTCA & 33 & 60 & 4 & $1(25)$ & 2.583 & 0.590 \\
\hline OPZ-11 & GGGTCTCGGT & 33 & 70 & 6 & $6(100)$ & 3.417 & 0.724 \\
\hline OPZ-12 & TCAACGGGAC & 34 & 60 & 2 & $2(100)$ & 1.333 & 0.889 \\
\hline OPL-19 & GAGTGGTGAC & 33 & 60 & 5 & $5(100)$ & 3.917 & 0.841 \\
\hline OPZ-13 & GACTAAGCCC & 33 & 60 & 8 & $8(100)$ & 4.167 & 0.911 \\
\hline OPZ-14 & TCGGAGGTTC & 34 & 60 & 5 & $2(100)$ & 2.500 & 0.907 \\
\hline \multirow[t]{2}{*}{ OPL-18 } & ACCACCCACC & 33 & 70 & 11 & $11(100)$ & 5.417 & 0.895 \\
\hline & & & & 264 & 255 (96.59) & & \\
\hline
\end{tabular}




\begin{tabular}{|c|c|c|c|c|c|c|c|}
\hline Primers & Sequence5'to3' & $\begin{array}{l}\mathbf{T m} \\
\left({ }^{\circ} \mathbf{C}\right)\end{array}$ & $\begin{array}{l}\text { GC } \\
(\%)\end{array}$ & $\begin{array}{c}\text { Total } \\
\text { bands }\end{array}$ & $\begin{array}{c}\text { Polymorphic } \\
\text { band }(\%)\end{array}$ & $\mathbf{R p}$ & PIC \\
\hline UBC880 & GGAGAGGAGAGGAGA & 55.6 & 60 & 14 & $14(100)$ & 6.083 & 0.862 \\
\hline UBC834 & AGAGAGAGAGAGAGACYT & 54.5 & 47 & 10 & $10(100)$ & 5.333 & 0.873 \\
\hline UBC856 & ACACACACACACACACCTA & 55.6 & 47 & 12 & $11(91.66)$ & 5.833 & 0.651 \\
\hline UBC810 & GAGAGAGAGAGAGAGAT & 54.5 & 47 & 10 & $10(100)$ & 4.167 & 0.727 \\
\hline UBC840 & GAGAGAGAGAGAGAGAYT & 57.5 & 47 & 9 & $9(100)$ & 5.333 & 0.881 \\
\hline UBC843 & CTCTCTCTCTCTCTCTRA & 53.2 & 47 & 9 & $9(100)$ & 4.083 & 0.906 \\
\hline UBC811 & GAGAGAGAGAGAGAGAC & 60.0 & 53 & 8 & $8(100)$ & 3.250 & 0.892 \\
\hline UBC888 & BDBCACACACACACACA & 60.0 & 50 & 12 & $12(100)$ & 4.917 & 0.802 \\
\hline UBC861 & ACCACCACCACCACCACC & 60.0 & 67 & 10 & $10(100)$ & 7.000 & 0.860 \\
\hline UBC835 & AGAGAGAGAGAGAGAGYC & 54.5 & 53 & 9 & $8(88.88)$ & 3.833 & 0.820 \\
\hline UBC825 & ACACACACACACACACT & 50.0 & 47 & 11 & $11(100)$ & 3.667 & 0.825 \\
\hline UBC866 & СТССТССТССТССТССТС & 54.5 & 67 & 10 & $10(100)$ & 5.667 & 0.732 \\
\hline UBC841 & GAGAGAGAGAGAGAGACTC & 57.5 & 53 & 9 & $9(100)$ & 4.750 & 0.802 \\
\hline UBC813 & СТСТСТСТСТСТСТСТT & 54.5 & 47 & 8 & $8(100)$ & 5.833 & 0.883 \\
\hline UBC887 & DVDTCTCTCTCTCTCTC & 60.0 & 50 & 7 & $6(85.71)$ & 4.833 & 0.794 \\
\hline UBC822 & TCTCTCTCTCTCTCTCA & 54.5 & 47 & 9 & $9(100)$ & 4.917 & 0.824 \\
\hline UBC892 & TAGATCTGATATCTGAATTCCC & 53.2 & 36 & 9 & $9(100)$ & 1.667 & 0.534 \\
\hline UBC855 & ACACACACACACACACCTT & 57.5 & 47 & 9 & $9(100)$ & 6.417 & 0.865 \\
\hline UBC884 & HBHAGAGAGAGAGAGAG & 53.2 & 50 & 5 & $5(100)$ & 2.833 & 0.828 \\
\hline UBC848 & CACACACACACACACAAGG & 57.5 & 53 & 7 & $7(100)$ & 9.500 & 0.731 \\
\hline UBC842 & GAGAGAGAGAGAGAGAYG & 60.0 & 53 & 8 & $8(100)$ & 7.083 & 0.783 \\
\hline UBC890 & VHVGTGTGTGTGTGTGT & 57.5 & 50 & 8 & $8(100)$ & 4.083 & 0.844 \\
\hline UBC824 & TCTCTCTCTCTCTCTCG & 54.5 & 53 & 6 & $6(100)$ & 5.083 & 0.787 \\
\hline UBC853 & TCTCTCTCTCTCTCTCRT & 55.6 & 47 & 7 & $7(100)$ & 6.250 & 0.781 \\
\hline $\mathrm{BC} 854$ & TCTCTCTCTCTCTCTCAGG & 57.5 & 53 & 6 & $6(100)$ & 3.500 & 0.869 \\
\hline UBC891 & HVHTGTGTGTGTGTGTG & 57.5 & 50 & 5 & $5(100)$ & 5.333 & 0.768 \\
\hline UBC809 & AGAGAGAGAGAGAGAGG & 57.5 & 53 & 5 & $5(100)$ & 5.250 & 0.712 \\
\hline UBC815 & СТСТСТСТСТСТСТCTG & 54.5 & 53 & 7 & $7(100)$ & 6.583 & 0.830 \\
\hline UBC836 & AGAGAGAGAGAGAGACYA & 54.5 & 47 & 7 & $7(100)$ & 5.500 & 0.867 \\
\hline BC850 & GTGTGTGTGTGTGTGTCTC & 60.0 & 53 & 5 & $5(100)$ & 4.500 & 0.720 \\
\hline UBC812 & GAGAGAGAGAGAGAGAA & 50.0 & 47 & 3 & $3(100)$ & 2.583 & 0.607 \\
\hline UBC814 & СТСТСТСТСТСТСТСТА & 54.5 & 47 & 6 & $6(100)$ & 5.417 & 0.562 \\
\hline UBC886 & VDVCTCTCTCTCTCTCT & 54.5 & 50 & 5 & $5(100)$ & 5.750 & 0.800 \\
\hline UBC844 & CTCTCTCTCTCTCTCTRG & 50.0 & 53 & 4 & $4(100)$ & 2.417 & 0.898 \\
\hline UBC895 & AGAGTTGGTAGCTCTTGATC & 57.5 & 45 & 4 & $4(100)$ & 4.250 & 0.898 \\
\hline
\end{tabular}

R-Wobble(A+G),H-Wobble(A+C+T),Y-Wobble(C+T),Y Q-3'Wobble(C+T),D-Wobble

$(\mathrm{A}+\mathrm{G}+\mathrm{T}), \mathrm{V}-\mathrm{Wobble}(\mathrm{A}+\mathrm{C}+\mathrm{G}), \mathrm{B}-\mathrm{Wobble}(\mathrm{C}+\mathrm{G}+\mathrm{T})$

Supplementary Table S4: DetailsofSSRprimers(Wangetal.,2010)selectedforthe study

\begin{tabular}{|c|c|c|c|c|c|c|c|}
\hline Primers & PrimerSequence(5'.........3') & $\begin{array}{c}\text { Tm } \\
\left({ }^{\circ} \mathbf{C}\right)\end{array}$ & $\begin{array}{c}\text { GC } \\
(\boldsymbol{\%})\end{array}$ & $\begin{array}{c}\text { Total } \\
\text { bands }\end{array}$ & $\begin{array}{c}\text { Polymorphic } \\
\text { bands( })\end{array}$ & Rp & PIC \\
\hline S24 & $\begin{array}{c}\text { F:GCTCTGCGTTTCATTCTTCA } \\
\text { R:TGAACCCTCAGACTCAAACTC }\end{array}$ & 60 & 46 & 5 & $4(80)$ & 3.250 & 0.961 \\
\hline N6 & $\begin{array}{c}\text { F:GGGAATTCTCAAAGAGCCAGA } \\
\text { R:TGGCACACTCTGCATGAAAT }\end{array}$ & 57 & 46 & 4 & $4(100)$ & 0.750 & 0.786 \\
\hline \multirow{2}{*}{ N24 } & $\begin{array}{c}\text { F:GCTCTGCGTTTCATTCTTCA } \\
\text { R:TGAACCCTCAGACTCAAACTC }\end{array}$ & 57 & 46 & 6 & $6(100)$ & 1.333 & 0.581 \\
\hline \multirow{2}{*}{ S13 } & $\begin{array}{c}\text { F:TTGGTTGTGGTGCTGAGTTC } \\
\text { R:GATGTAGGGGTTGGGTTGAT }\end{array}$ & 57 & 50 & 5 & $5(100)$ & 3.917 & 0.864 \\
\hline & F:GTCTTCCAGGTTGGGAACAG & 59 & 53 & 10 & $10(100)$ & 0.653 & 0.859 \\
\hline
\end{tabular}




\begin{tabular}{|c|c|c|c|c|c|c|c|}
\hline $\mathrm{N} 1$ & R: ATCTGGTTCCTCGGGAGATT & & & & & & \\
\hline S15 & $\begin{array}{c}\text { F:GGGTAGTGGAATGATGGGTT } \\
\text { R:TAGTGTTTTCGTGAGGGAGG }\end{array}$ & 57 & 50 & 5 & $5(100)$ & 1.917 & 0.886 \\
\hline S33 & $\begin{array}{c}\text { F: ATTTAGTGGGGCGGGTAGT } \\
\text { R:TGGATGAGCATGTTAGGGATC }\end{array}$ & 54 & 50 & 4 & $4(100)$ & 2.500 & 0.701 \\
N9 & $\begin{array}{c}\text { F: ATCCATCCCCACAAGTTGAA } \\
\text { R:CCATAAGGATATGTTTGCATGG }\end{array}$ & 60 & 43 & 2 & $1(50)$ & 0.833 & 0.413 \\
\hline S18 & $\begin{array}{c}\text { F:GGGTAGTGGAATGATGGGTT } \\
\text { R:TAGTGTTTTCGTGAGGGAGG }\end{array}$ & 58 & 50 & 3 & $3(100)$ & 1.667 & 0.825 \\
\hline S32 & $\begin{array}{c}\text { F:CTAAATCACGCAAACCCATC } \\
\text { R:GAGCAAAAGACTGAGGAAAACT }\end{array}$ & 54 & 43 & 4 & $4(100)$ & 3.250 & 0.891 \\
\hline S9 & $\begin{array}{c}\text { F:TTCCCATTCACAGATCACTCC } \\
\text { R:CCACCAAATTCAAGAACCCAC }\end{array}$ & 57 & 48 & 2 & $2(100)$ & 1.500 & 0.734 \\
\hline N5 & $\begin{array}{c}\text { F:CGTCGCTCTCACAAGAGATAAG } \\
\text { R:TTTGGTGGAAATCCCCTATT }\end{array}$ & 59 & 45 & 2 & $2(100)$ & 1.333 & 0.722 \\
\hline S20 & $\begin{array}{c}\text { F:CCCCTTCTAATCACAACCAA } \\
\text { R:GGCCTAATTTCTGCCCTTT }\end{array}$ & 58 & 46 & 3 & $3(100)$ & 1.917 & 0.886 \\
\hline N12 & $\begin{array}{c}\text { F:CAGAGGGGTGGTTCCTCTTT } \\
\text { R:CCACATGGATGATCGAGAGA }\end{array}$ & 59 & 53 & 3 & $3(100)$ & 2.250 & 0.920 \\
\hline S12 & $\begin{array}{c}\text { F:CAGAGGGGTGGTTCCTCTTT } \\
\text { R:CCACATGGATGATCGAGAGA }\end{array}$ & 57 & 53 & 3 & $3(100)$ & 2.083 & 0.814 \\
\hline S26 & $\begin{array}{c}\text { F:GAACGCCCTGTGACTTTAGC } \\
\text { R:TTTCGTCTTCCAATGAGCC }\end{array}$ & 58 & 51 & 2 & $2(100)$ & 2.750 & 0.829 \\
\hline & & & & $\mathbf{6 3}$ & $\mathbf{6 1 ( 9 5 . 6 2 )}$ & $\mathbf{1 . 9 9 3}$ & $\mathbf{0 . 7 9 2}$ \\
\hline
\end{tabular}

Supplementary TableS5: Nei'scoefficientfor geneticdiversity(abovediagnol)andNeiand Licoefficientfor geneticdistance (belowdiagnol)amongthespecies.

\begin{tabular}{|l|l|l|l|l|}
\hline & M.charantia & M.cochinchinensis & M.dioica & M.balsamina \\
\hline M.charantia & --- & 0.543 & 0.5728 & 0.7085 \\
\hline M.cochinchinensis & 0.6107 & --- & 0.7362 & 0.6924 \\
\hline M.dioica & 0.5572 & 0.3111 & -- & 0.7062 \\
\hline M.balsamina & 0.3446 & 0.3675 & 0.3479 & --- \\
\hline
\end{tabular}

Supplementary Table S6:Primerswithspecies-specificbandswiththeirmolecularsize

\begin{tabular}{|c|c|}
\hline Uniquemarkers & Momordicaspeciesidentified with molecularsize $(b p)$ \\
\hline \multicolumn{2}{|r|}{ RAPDPrimers } \\
\hline OPM-14 & M.dioica(650), M.cochinchinensis $(900)$ \\
\hline OPL-07 & M.dioica $(950)$ \\
\hline OPL-08 & M.cochinchinensis $(850)$ \\
\hline OPAF-12 & M.charantia $(400)$ \\
\hline OPH-10 & M.dioica(750), M.charantia $(400)$ \\
\hline OPAG-012 & M.charantia(500), M.cochinchinensis $(950)$ \\
\hline OPAF-04 & M.cochinchinensis $(890)$ \\
\hline OPAF-11 & M.charantia(1250), M.dioica(350) \\
\hline OPC-16 & M.charantia $(900)$ \\
\hline OPC-08 & M.charantia $(850)$ \\
\hline OPZ-11 & M.balsamina $(1300)$ \\
\hline \multicolumn{2}{|r|}{ SSRsPrimers } \\
\hline N01 & M.charantia $(200,250)$ \\
\hline $\mathrm{N} 24$ & M.charantia $(320)$ \\
\hline S13 & M.charantiaandM.cochinchinensis(220) \\
\hline S32 & M.charantia $(500)$ \\
\hline S18 & M.dioica(240) \\
\hline
\end{tabular}




\begin{tabular}{|c|c|}
\hline S33 & M.dioica $(425)$ \\
\hline S33 & M.charantiaandM.balsamina $(380)$ \\
\hline S33 & M.cochinchinensisand M.dioica $(250)$ \\
\hline & ISSRMarkers \\
\hline UBC866 & M.charantia $(700,1300)$, M.dioica $(800)$, M.charantiaandM.cochinchinensis $(550)$ \\
\hline UBC822 & $\begin{array}{c}\text { M.charantia(700,900,1400), M.dioica }(1100), \text { M.cochinchinensis }(330,400,800), \\
\text { M.charantiaandM.dioica }(550)\end{array}$ \\
\hline UBC834 & M.dioica $(1500)$, M.charantia $(260,275,500)$, M.cochinchinensis $(250)$ \\
\hline UBC887 & $\begin{array}{c}\text { M.charantia }(1500), \text { M.dioica }(700,750,1200), \text { M.cochinchinensis }(700), \\
\text { M.dioica } a n d \text { M.balsamina }(500)\end{array}$ \\
\hline UBC842 & $\begin{array}{c}\text { M.charantia(500,680,1200,1500), M.dioica(500,750), M.cochinchinensis }(700), \\
\text { M.charantiaandM.balsamina }(480)\end{array}$ \\
\hline UBC836 & $\begin{array}{c}\text { M.dioica(1000), M.cochinchinensis }(540,800), \text { M.dioicaandM.balsamina }(275,500), \\
\text { M.balsamina }(260)\end{array}$ \\
\hline UBC886 & $\begin{array}{c}\text { M.charantiaandM.balsamina(1000), M.dioica(750,900), M.cochinchinensis }(600), \\
\text { M.charantia }(500)\end{array}$ \\
\hline UBC888 & M.charantia(2400), M.cochinchinensis(250,2000), M.dioica(250,1000,1700) \\
\hline UBC890 & M.cochinchinensis $(500,1500)$, M.dioica $(660)$, M.balsamina $(430)$ \\
\hline UBC840 & M.charantiaandM.cochinchinensis $(250)$ \\
\hline UBC855 & M.charantia(2500), M.cochinchinensis(575) \\
\hline UBC841 & M.cochinchinensis(280), M.dioica(250) \\
\hline UBC848 & M.charantiaand M.dioica(1500), M.cochinchinensisandM.dioica $(900)$, M.dioica $(500)$ \\
\hline UBC812 & M.cochinchinensisand M.balsamina $(300)$ \\
\hline UBC825 & $\begin{array}{c}\text { M.balsamina(590,1270), M.charantia(530,1100), M.cochinchinensisandM.dioica(645), } \\
\text { M.dioica }(600)\end{array}$ \\
\hline UBC861 & M.dioica(1400), M.cochinchinensis $(300,400,750,900)$, M.dioica $(300,335)$ \\
\hline UBC892 & M.cochinchinensisandM.dioica $(300,1600)$ \\
\hline UBC843 & M.dioica(800), M.cochinchinensisandM.dioica(400) \\
\hline UBC884 & M.charantia $(200,600)$, M.charantiaandM.dioica $(530)$ \\
\hline UBC855 & M.cochinchinensisandM.dioica $(200,700,750)$, M.charantia $(450)$, M.cochinchinensis $(300)$ \\
\hline UBC891 & M.charantia $(250)$ \\
\hline UBC815 & M.dioica $(800,1300)$, M.charantia $(900,1200)$ \\
\hline UBC809 & $\begin{array}{c}\text { M.cochinchinensis }(600), \text { M.charantia, M.dioicaand } \\
\text { M.balsamina }(425,500), \text { M.dioica }(300)\end{array}$ \\
\hline UBC891 & M.cochinchinensisandM.dioica(750), M.cochinchinensis(300) \\
\hline UBC844 & M.charantia $(900)$, M.cochinchinensisandM.dioica $(500)$ \\
\hline UBC880 & M.dioica(1300), M.charantia $(335,750)$, M.cochinchinensis $(573)$ \\
\hline UBC856 & M.cochinchinensis $(300,1300)$, M.dioica $(250)$, M.charantiaandM.balsamina $(240)$ \\
\hline UBC853 & M.charantiaandM.balsamina(1500), M.dioica $(750,1100)$, M.cochinchinensis $(500)$ \\
\hline UBC813 & M.dioica(1300), M.cochinchinensis $(1000)$, M.charantia(400) \\
\hline UBC810 & M.cochinchinensis(1200), M.charantia(250,275,730), M.balsamina(250) \\
\hline UBC814 & M.cochinchinensisandM.dioica $(400)$ \\
\hline UBC835 & M.charantiaandM.balsamina $(200,1000)$, M.charantia(600), M.dioica(250) \\
\hline UBC811 & $\begin{array}{l}\text { M.charantia(1600), M.cochinchinensisandM.dioica }(1500), \text { M.charantiaand } \\
\text { M.dioica }(300,635), \text { M.dioica }(700)\end{array}$ \\
\hline
\end{tabular}

\section{Acknowledgement}

The authors are highly acknowledged and express their gratitude to high supportof theDirector,IndianInstituteofVegetableResearch (ICAR), Varanasiforproviding necessary laboratoryfacilitiesto smoothly facilitate the work.

\section{Funding/Support}

The present work is financially supported byIndian Institute of Vegetable Research, Post Box 01, Jakhini (Shahanshahpur), Varanasi, Uttar Pradesh 221 305, India 


\section{Conflict of Interest}

The authors declared no conflict of interest.

\section{References}

1. Shukla, A., Singh, V.K., Bharadwaj, D.R., Kumar, R., Rai, A., Rai, A.K., Mugasimangalam, R., Parameswaran, S., Singh, M., Naik, P.S., 2015. De Novo Assembly of Bitter Gourd Transcriptomes: Gene Expression and Sequence Variations in Gynoecious and Monoecious Lines. PLoS ONE 10 (6), e0128331. doi:10.1371/journal.pone.0128331.

2. Baranski,R.,Grzebelus,D., Frese,L.,2001.Evaluation ofgeneticdiversityinagardenbeetgermplasmcollection usingRAPDtechnique.ActaHorticulturae.546,165-170.

3. Behera,T.K.,Singh,A.K.,Staub,J.E.,2008.Comparativeanalysis ofgeneticdiversity inIndianbittergourd (MomordicacharantiaL.)usingRAPDandISSRmarkersfordevelopingcropimprovement strategies. ScientiaHorticulturae115, 209-217.

4. Bharathi,L.K.,Munshi,A.D., Behera,T.K., Vinod.,John,K.J.,Das,A.B., Bhat,K.V.,Sidhu,A.S.,2012.Productionand preliminary characterizationoninter-specific hybridsderivedfromMomordicaspecies.CurrentScience 103,178-186.

5. Bharathi,L.K.,Parida,S.K.,Munshi, A.D.,Behera,T.K.,Raman,K.V.,Mohapatra,T.,2012. Moleculardiversityand phoneticrelationshipofMomordicaspp.ofIndianoccurrence.GeneticResourcesandCropEvolution 59,937-948.

6. Cai,Y.L.,Cao,D.W.,Zhao,G.F.,2007.StudiesongeneticvariationincherrygermplasmusingRAPDanalysis. ScientiaHorticulturae111,248-254.

7. Dalamu, T.K.B., Gaikwad, A.B., Saxena, S., Bharadwaj, C., Munshi, A.D., 2012. Morphological and molecular analyses define the genetic diversity of Asian bitter gourd (Momordicacharantia, L.). Australian Journal of Crop Science 6, 261-267.

8. Dey,S.S.,Singh,A.K.,Chandel,D.,Behera,T.K.,2006.Geneticdiversityofbittergourd(MomordicacharantiaL.) genotypesrevealedbyRAPDmarkersandagronomictraits.ScientiaHorticulturae109,21-28.

9. Doyle,J.J., Doyle,J.L.,1990.ArapidDNAisolationprocedurefromsmallquantitiesoffreshleaftissue. PhytochemBulletin.119,11-15.

10. Dutta,P.K.,Chakravarty,A.K.,Chowdhary,U.S.,1981.Vicine,afavism-inducingtoxinfromMomordicacharantiaL., seeds.IndianJournalofChemistry20,669-671.

11. Fenwick,A.L.,Ward, S.M.,2001.UseofrandomamplifiedpolymorphicDNA markersforcultivaridentificationin mint.HortScience 36,761-764.

12. Gaikwad,A.B.,Behera,T.K.,Singh,A.K.,Chandel,D.,Karihaloo, J.L.,Staub, J.E.,2008.AFLPanalysis provides strategiesforimprovementofbittergourd(MomordicacharantiaL.).HortScience43,127-133.

13. Gillies,A.C.M.,Cornelius, J.P.,Newton,A.C.,Navarro,C.,Hernandez, M.,Wilson,J.,1997.GeneticvariationinCosta Ricanpopulations ofthetropicaltimberspecies CedrelaodorataL.assessedusingRAPDs. Molecular Ecology6,1133-1146.

14. Grover, J.K., Yadav, S., Vats, V., 2002.Medicinal plantsof India with antidiabetic potential. Journal ofEthnopharmacology81,81-100.

15. Gwanama,C.,Labuschagne, M.T.,Botha,A.M.,2000.Analysis ofgeneticvariationinCucurbita moschataby randomamplifiedpolymorphicDNA(RAPD) markers. Euphytica113,19-24.

16. Hooker,J.D., 1879.TheFloraofBritishIndia(reprint1961),Vol.2. L. ReeveCo.Kent.,England.

17. Iqbal, M., Munawar, M., Najeebullah, M., Din, A., 2016. Assessment of genetic diversity in bitter gourd. International Journal of Vegetable Science. DOI:10.1080/19315260.2015.1107674.

18. Jaccard,P.,1908.Nouvellesrecherchessurladistributionflorale. Bull.Soc.VanddoiseSci.Nat.44,223-270.

19. Ji,Y.,Luo,Y.,Hou,B.,Wang,W.,Zhao,J.,Yang,L.,Xue,Q., microsatellitelociinMomordicacharantia(Cucurbitaceae)

Ding,X.,2012.Developmentofpolymorphic andtheirtransferabilitytoothercucurbit species.ScientiaHorticulturae140,115-118.

20. John,K.J.,Antony, V.T.

Roy,Y.C.,2007.On

the occurrence, distributionandtaxonomyofMomordicasubangulataBlumesubsp.renigera(G.Don)deWildeinIndia.GeneticR esourcesandCropEvolution54,1327-1332.

21. John,K.J.,Antony,V.T.,2008.PreliminaryObservationsonFruitRipening,PredationandSeedDispersalinthe WildMomordicaSpecies.JournalofPlantGeneticResources21,128-131.

22. Karp,A.,Edwards, K.J.,1997.Molecular techniquesinanalysisoftheextentanddistributionofgenetic diversity. In:Moleculargenetictechniquesforplantgeneticresources,Ayad,W.G.,Hodgkin,T.,Jaradat,A., IPGRI,Rome.pp11-22.

23. Kumar,G.,Mahajan,R.K.,Sapra,R.L.,Gangopadhyay,K.K.,Meena,B.L.,Tiwari,S.K.,Mishra,S.K.,Sharma,S.K.,2010. Development ofcoresetinbrinjal(SolanummelongenaL.)withlimitedtraits.IndianJournalofPlant GeneticResources.22,206-210.

24. Kundu, B.C., Hosssain, M.M., Khalequemian., Mian, I.H., 2012. Genetic divergence in bitter gourd (MomordicacharantiaL.) Journal of Asiatic Society Bangladesh Science 38(2), 125-134.

25. Lowe,A.J.,Hanotte,O.,Guarino,L.,1996.Standardizationofmolecular genetictechniques forthecharacterization ofgermplasmcollections:ThecaseforRandomAmplifiedPolymorphic

DNA(RAPD).PlantGenetic 
ResourcecNewsletter107,50-54.

26. Maguire,T.L.,Collins,G.,Sedgley,M.,1994.AmodifiedCTABDNAextractionprocedureforplantsbelongingto familyProtesceae.PlantMolecularBiologyReporter12,106-109.

27. Martin,J.P.,Bermejo,J.E.H.,2000.GeneticvariationintheendemicandendangeredRosmarinustomentosusHuberMoarthandMarine(Labiatae)usingRAPDmarkers.JournalofHeredity85,434-443.

28. Nei, M., Li, W.H., 1979. Mathematical modelfor studying genetic variation in terms of restriction endonucleases.ProceedingsoftheNationalAcademyofSciences,USA76,5269-5273.

29. Page, R.D.M.,1996.TREEVIEW:Anapplicationto displayphylogenetictreesincomputers.Computerapplicationin Bioscience12,357-378.

30. Pavlicek,A.,Hrda,S.,Flegr,J.,1999.Freewareprogram forconstructionofphylogenetic treeonbasisofdistance dataandbootstrap/jack-knife genusfrenkelia.FoliaBiologica45,97-99.

31. Pradeepkumar,T.,Karihaloo,J.L.,Archak,S.,Baldev,A.,2003.AnalysisofgeneticdiversityinPipernigrumL. usingRAPDmarkers.GeneticResourcesandCropEvolution50,469-475.

32. Raina,S.N.,Rani,V.,Kojima,T.,Ogihara,Y.,Singh,K.P.,Devarumath, usefulgeneticmarkersforanalysisofgeneticdiversity, varietalidentification relationshipinpeanut(ArachishypogiaL)cultivarsandwildspecies.Genome44,763-772.

R.M.,2001.RAPDandISSRfingerprintsas andphylogenetic

33. Raj,M.,Prasanna,N.K.P., $\quad$ Peter,K.V.,1993.BittergourdMomordicassp.In:Kalloo,G.,Berg,B.O.(Eds.),Genetic ImprovementofVegetableCrops.PergmonPress,Oxford,UK,pp.239-246.

34. Ram,D.,Kumar, S.,Banerjee,M.K.,Kalloo,G.,2002.Occurrence,identificationandpreliminarycharacterizationof gynoecisminbittergourd.IndianJournalofAgriculturalScience72,348-349.

35. Rashid,M.M.,1993.ShabjiBijgan,2nded. RashidPublishingHouse,Banani,Dhaka(inBengali).

36. Rasul,M.G., 2003.Studyonparthenocapyandgeneticdivergenceinkakrol(MomordicadioicaRoxb.).Ph.D.Thesis. KyushuUniversity,Fukuoka,Japan.

37. Rasul, M.G., Hamamatsu,M., Okubo, H., 2007. Genetic relatedness(diversity)and cultivaridentificationby randomlyamplifiedpolymorphic DNA (RAPD)markersinteaslegourd(MomordicadioicaRoxb.). ScientiaHorticulturae111,271-279.

38. Robinson,R.W.,Decker-Walters,D.S.,1999.Cucurbits.CABInternational.Wallingford,Oxford,UK,226.

39. Schaefer,H.,Renner,S.S.,2010. Athree-genomephylogenyofMomordica(Cucurbetecea)suggests sevenreturns fromdioecyto monoecyand recent long-distancedispersalto Asia. MolecularPhylogeneticsand Evolution54,553-560.

40. Singh,A.K.,1990.Cytogenetics andevolutioninthecucurbitaceae. In:Bates,D.M.,Robinson,R.W.,Jaffrey,C. (Eds.),BiologyandUtilizationofCucurbitaceae.CornellUniversityPress,NewYork,USA,pp.10-28.

41. Singh,A.K.,Behera, T.K.,Chandel,D.,Sharama,P.,Singh,N.K.,2007.Assessing geneticrelationshipamongbitter gourd(MomordicacharantiaL.)accessionusingintersimplesequencerepeat(ISSR)markers.Journal ofHorticultureScienceand.Biotechnology82,217-222.

42. Singh,A.K.,Smart, J.,Simpson,C.E.,Raina,S.N.,1998.Geneticvariationvis-à-vismolecularpolymorphism in groundnut,ArchishypogaeaL.,GeneticResourcesand CropEvolution45,119-126.

43. Singh, B., Singh, AK., Kumar, S., 2013. Genetic divergence studies in bitter gourd (M. charantia L). Academic Journal of Plant Sciences 6, 89-91.

44. Tiwari,S.K.,Karihaloo,J.L.,Hameed,N.,Gaikwad,A.B.,2009.Molecularcharacterization ofbrinjal(SolanummelongenaL.)cultivarsusingRAPDandISSRmarkers.JournalofPlantBiochemistry and Biotechnology18,189-195.

45. Trivedi,R.N., Roy,R.P., 1972.Cytologicalstudiesin some speciesofMomordica.Genetica43,282-291.

46. Ulanowsky,S.,Gogorcena,Y.,Martinez-de-Toda,F.,Borrege,J.,Ibanez,J.,Ortiz,J.M.,2001.Characterizationof GrapevineaccessionsatgermplasmbankwithRAPDandmicrosatellitemarkers.ActaHorticulturae546,271-280.

47. Wang,S.Z.,Pan,L.,Hu,K.,You,C.C.,Yi,D.,2010.Developmentandcharacterizationofpolymorphic microsatellite markersinMomordicacharantia(Cucurbitaceae).AmericanJournalofBotanydoi:10.3732:e75-e78.

48. Williams,J.G.K.,Kubelic,A.R.,Livak,K.J.,Rafalski,J.A.,Tingey,S.V.,1990.DNApolymorphisms amplifiedby arbitraryprimersareusefulasgeneticmarkers.Nucleic Acid Research18,6531-6653.

49. Yeh,G.Y.,Eisenber,D.M.,Kaptchuk, T.J., PhillipsR.S.,2003.Systematicreviewof herbsanddietarysupplementsfor glycemiccontrolindiabetes.DiabetesCare26,1277-1294. 